University of San Diego

Digital USD

2008-10-16

\title{
The Use of Healing Touch in Adults with Hepatitis C Virus Receiving Interferon and Ribavirin: A Pilot Study
}

Margaret Ann Taylor PhD, MSN, CNS, HTCP/I

University of San Diego

Follow this and additional works at: https://digital.sandiego.edu/dissertations

Part of the Nursing Commons

\section{Digital USD Citation}

Taylor, Margaret Ann PhD, MSN, CNS, HTCP/I, "The Use of Healing Touch in Adults with Hepatitis C Virus Receiving Interferon and Ribavirin: A Pilot Study" (2008). Dissertations. 377.

https://digital.sandiego.edu/dissertations/377

This Dissertation: Open Access is brought to you for free and open access by the Theses and Dissertations at Digital USD. It has been accepted for inclusion in Dissertations by an authorized administrator of Digital USD. For more information, please contact digital@sandiego.edu. 
UNIVERSITY OF SAN DIEGO

Hahn School of Nursing and Health Science

DOCTOR OF PHILOSOPHY IN NURSING

THE USE OF HEALING TOUCH IN ADULTS WITH HEPATITIS C VIRUS

RECEIVING INTERFERON AND RIBIVIRAN: A PILOT STUDY

by

Margaret Ann Taylor, MSN, CNS, HTCP/I

A dissertation presented to

THE FACULTY OF THE HAHN SCHOOL OF NURSING AND HEALTH SCIENCE

UNIVERSITY OF SAN DIEGO

\author{
In partial fulfillment of the \\ requirements for the degree \\ DOCTOR IN PHILOSOPHY OF NURSING \\ October 16, 2008 \\ Cynthia D. Connelly, PhD, RN, FAAN (Chair) \\ Linda Robinson, $\mathrm{PhD}, \mathrm{RN}$ \\ Kevork M. Peltekian, MD, FRCPC \\ Jane M. Georges, $\mathrm{PhD}, \mathrm{RN}$
}


C Copyright 2009

Margaret Ann Taylor

All Rights Reserved 


\section{Dedication}

To my parents

Jeanne Seymour Bairos and William Alexander Taylor

and

my three sons

Alan Sweet, Jamie Sweet, and Andy Sweet. 


\section{Acknowledgements}

I wish to thank Dr. Cynthia Connelly for her mentorship, advice and support throughout this work.

I wish to thank Dr. Linda Robinson for her support and gracious patience throughout the $\mathrm{PhD}$ program and the completion of this work. I am especially grateful for her gifted insight, excellent editing skills and unending encouragement that provided the motivation I needed to complete this process.

I am indebted to Dr. Kevork Peltekian for his guidance, support and sharing of his expert knowledge of Hepatitis C. I am grateful for his efforts that led to access of QEII Capital Health Victoria General as the site for this project.

Thank you to Dr. Georges who was instrumental in assistance with the IRB process and providing the support I needed to complete this process.

I would also like to express my gratitude to the following colleagues who each provided their own unique assistance that contributed to the completion of this process: Ann Peltekian, RN, BSN, Geri Hirsh, RN, MSN/NP, Carla Burgess, RN, MSN/NP, Mary MacAdam, RN BSN, and Margaret Archibald RN, BSN.

In addition, I wish to thank Dr. Donna Agan for her unfailing support throughout the completion of this work. 


\section{Table of Contents}

Page

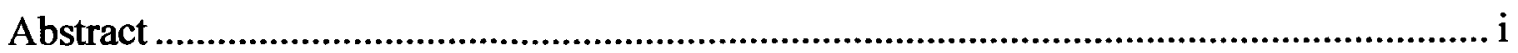

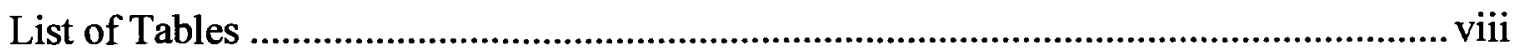

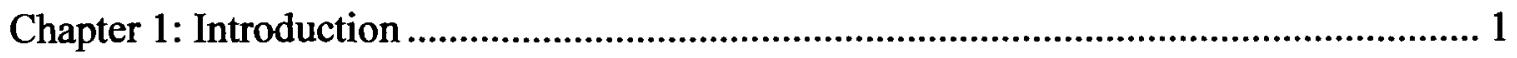

Background and Significance ......................................................................... 1

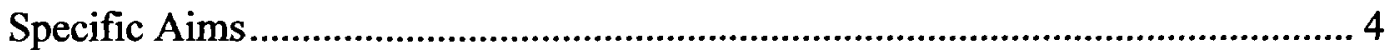

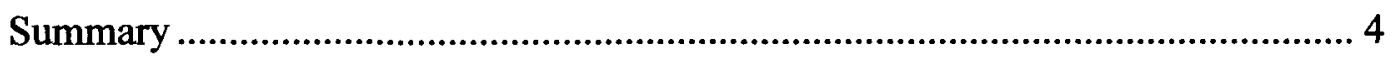

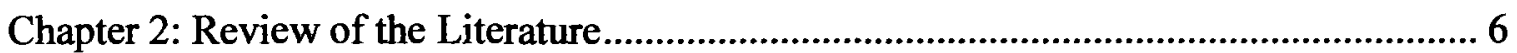

Rogers Science of Unitary Human Beings ........................................................... 6

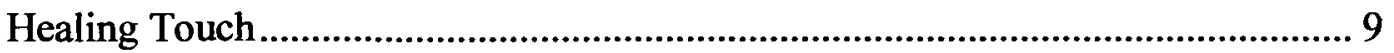

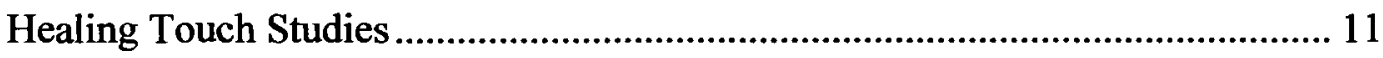

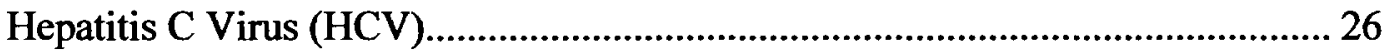

Chronic Hepatitis C and Pegylated Interferon and Ribavirin Treatment.............. 27

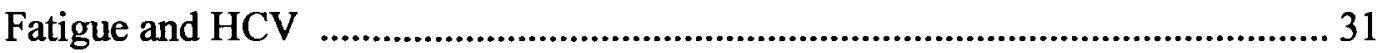

Summary

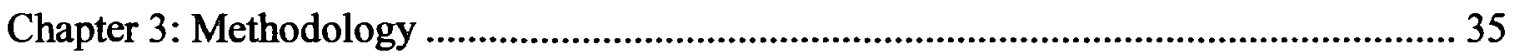

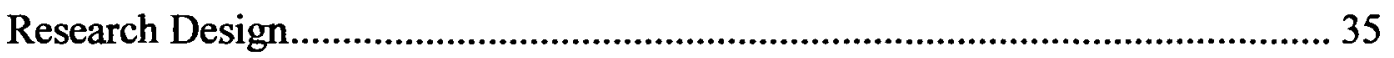

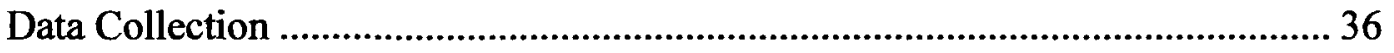

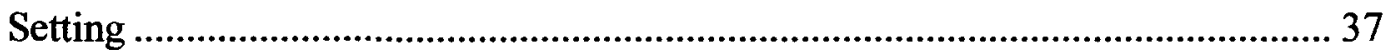

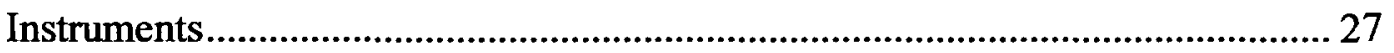

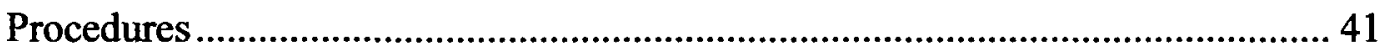

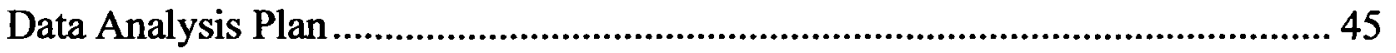


Protection of Human Subjects.

An Overview of Subject Selection and Characteristics .............................. 46

Inclusion of Women, Minorities, and Children ............................................ 47

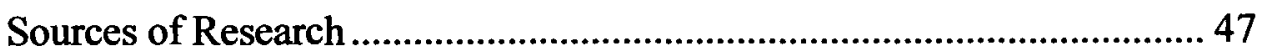

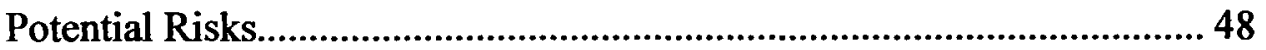

Risk Management Procedures.................................................................... 48

Potential Benefits/Compensation .............................................................. 48

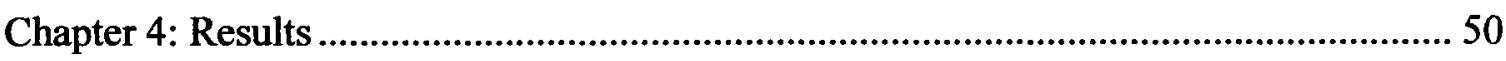

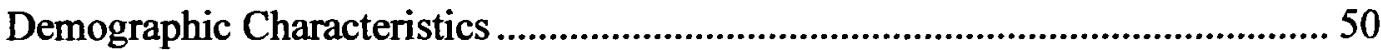

Comparison of Groups at Baseline ................................................................... 53

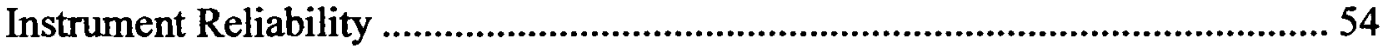

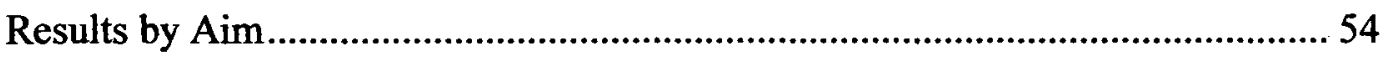

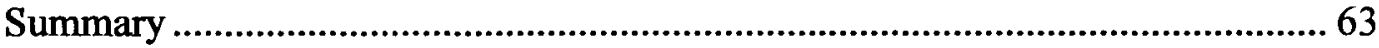

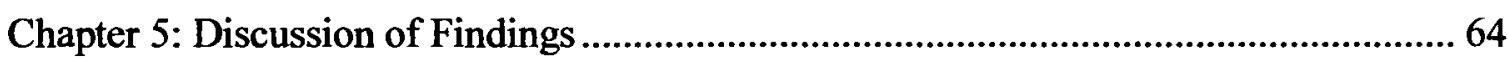

Issues Regarding Methodology and Results .....................................................64

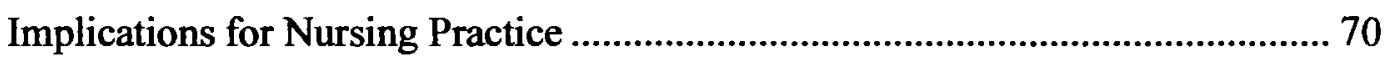

Implications for Nursing Education ..................................................................... 71

Implications for Nursing Research ................................................................. 71

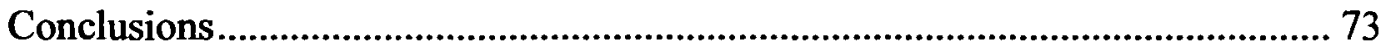

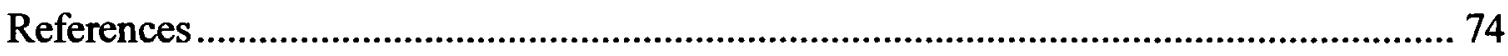

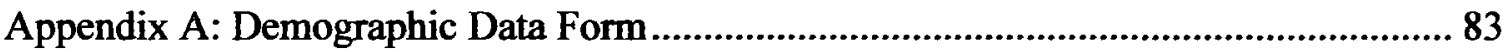

Appendix B: Healing Touch Magnetic Clearing Intervention.......................................... 84

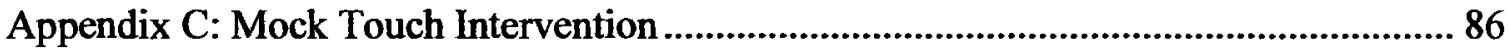


Appendix D: Schwartz Cancer Fatigue Scale ................................................................. 88

Appendix E: Subjects Co-morbidities, Hemoglobin Level, and Current Meds................ 89

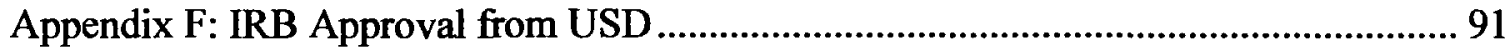

Appendix G: Research Ethics Board Approval Capital Health......................92 


\section{List of Tables}

Tables

1. Contraindication for Starting Combination Interferon and Ribavirin Treatment . 28

2. Drugs Used in the Treatment of Chronic Hepatitis C Virus 29

3. Experimental Randomized Controlled Design with Repeated Measures

4. Theoretical Construct and Empirical Linkages............................................... 38

5. Data Collection Procedures...................................................................... 45

6. Comparison of Demographic Variables of HT and MT groups ........................ 52

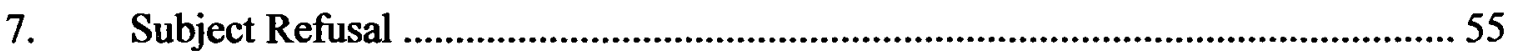

8. Pre-Post Fatigue Impact Scale (FIS) Scores ........................................... 58

9. Differences in Program Completers and Non-Completers in the FIS ............... 59

10. Schwartz Cancer Fatigue Scale (SCFS) Descriptive Statistics for Overall Group

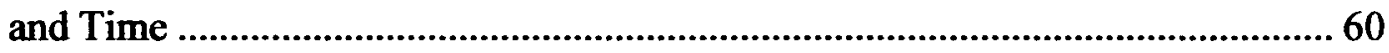

11. FIS Comparison of Domains Between Groups by Time .................................. 61

12. SCFS Descriptive Statistics by Group and Time ........................................ 62

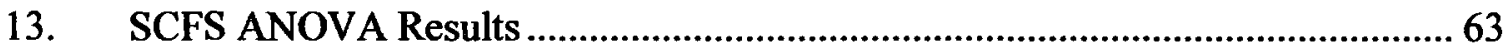




\section{Chapter 1}

\section{Introduction}

The purpose of this study was to test a protocol using a Healing Touch (HT) intervention to relieve fatigue experienced by persons with chronic hepatitis $\mathrm{C}$ virus (HCV) who were receiving a combination treatment of pegylated (PEG) interferon and ribavirin. The theoretical framework for the study was drawn from Martha Rogers' conceptual system, the science of unitary human beings (Rogers, 1970). This chapter will introduce the problem, present the background and significance, and specific aims for this study.

\section{Background and Significance}

Hepatitis $\mathrm{C}$ virus (HCV) is a major public health problem that has reached epidemic proportions with over 170 million people worldwide and 4.1 million individuals in the United States (US) having been exposed. Of these, 3.2 million (75-85\%) will develop a chronic $\mathrm{HCV}$ infection and an additional 19 thousand new cases will be diagnosed annually in the US. Hepatitis C virus is the most common blood borne viral infection and the leading cause of chronic liver disease and leading indication for liver transplant in adults in the US. This virus is ranked in the top the ten causes of death in all male adults as well as the number one cause of deaths among persons with HIV/AIDS (CDC, March 2008). 
At highest risk for $\mathrm{HCV}$ infection are persons with a history of intravenous drug use or those who received a blood transfusion or solid body transplant prior to 1992 when blood-screening methods became available. Although contaminated blood is the primary mode of transmission, $\mathrm{HCV}$ can be sexually transmitted and survive outside the body for as long as 14 days. The HCV progresses asymptomatically, slowly and often without detection over 2 to 4 decades, resulting in serious liver damage that leads to possible cirrhosis and carcinoma (Strader, Wright, Thomas \& Seeff, 2004; WHO 2002).

There is no vaccine available for $\mathrm{HCV}$ and until recently this disease was untreatable. Presently the only known cure for $\mathrm{HCV}$ is treatment with a combination of pegylated interferon and ribavirin; however, the related side effects are significant (CDC, 2008). There are reports of serious fatigue ranging from $56 \%$ to $92 \%$ of patients receiving this treatment (CDC 2008, Glacken, Coates, Kernohan, \& Hegarty, 2003; Schaefer, 2004; Zucker, 2001). Consequences of fatigue result in reduced or missed doses and lack of completion of the required 24 to 48 weeks of pharmacological treatment. In patients receiving a combination of pegylated interferon and ribavirin, the antiviral response is significantly improved with maintained adherence to the prescribed treatment (Friedrich-Rust, 2007; Zic, 2005). Finding effective non-pharmacological complementary and alternative medical therapy (CAM) interventions to prevent or manage fatigue in the chronic $\mathrm{HCV}$ population is important to support patients adherence to the current treatment for the best chance for a cure.

The National Center for Complementary and Alternative Medicine (NCCAM), a branch of the National Institutes of Health, reported that interest and use of (CAM) rose during the last decade in the US. Sixty-two percent of American adults reported having 
used at least one CAM therapy. Office visits to CAM providers out numbered those to primary care physicians during the same period. This represented a total expenditure of $\$ 30$ billion with $\$ 21.2$ billion of this amount being out-of-pocket payments (Eisenberg et al., 1998). The Institute of Medicine recommends that CAM treatments be held to the same standards of evidenced-based practice as conventional medical practices (IOM, 2005). Despite widespread use of CAM, limited evidence of clinical efficacy for these therapies has been provided by scientific evidence.

Healing Touch includes a group of non-invasive energy-based interventions that are increasingly being used as an adjunct to standard medical treatment in clinical practice throughout North America. This therapy promotes the potential for self-healing and the restoration of balance of the person's entire energy system (Healing Touch Notebook, 2007).

Although there are anecdotal clinical reports of the positive benefits of HT by practitioners and clients, only a small number of studies of HT for a variety of symptoms exist (Wardell \& Weymouth, 2004). These interventions require further theoretical development with adequate empirical support, documented underpinnings, and published research to scientifically validate their efficacy. An exhaustive review of the empirical literature found no study investigating HT for use in patients diagnosed with any liver disease. This is the first study, to our knowledge, to test the feasibility of a protocol for the effectiveness of HT as an intervention for symptom management in patients diagnosed with HCV.

This study is significant for nursing because HT is suggested as an evidencedbased nursing intervention recommended for use with the North American Nursing 
Diagnosis Association's label, disturbed energy field (Ackley, 2008). Nurse researchers have a responsibility to establish scientific evidence of nursing interventions.

Examination of the value and limitation of $\mathrm{HT}$ are needed to build the scientific base necessary to responsibly guide nursing policy and practice. The purpose of this study is to provide preliminary data by assessing the feasibility of a HT protocol for use in patients with $\mathrm{HCV}$ who presently were being treated with interferon and ribavirin.

Specific Aims

The aim of this study was to investigate a protocol using Healing Touch to decrease fatigue in patients with chronic $\mathrm{HCV}$ who were being treated with a combination of interferon and ribavirin when compared to a similar group of patients who received a mock touch intervention over three consecutive weeks. The specific aims of this study were to:

a) Determine the acceptance rate of all possible participants for study inclusion.

b) Determine the attrition rate of all participants enrolled in the study.

c) Describe fatigue over four weeks in patients with $\mathrm{HCV}$ receiving interferon and ribavirin.

d) Compare the level of fatigue in the control and experimental groups.

\section{Summary}

Hepatitis $\mathrm{C}$ is a serious viral epidemic in the US. A lengthy treatment of interferon and ribavirin is the only possible way to obtain a cure for individuals infected with chronic HCV. Many of these clients do not adhere or complete this treatment because of the significant side effects. Fatigue is reported as the most common and severe side effect of this combination treatment in patients with chronic HCV. Few effective interventions are 
available to treat fatigue in this $\mathrm{HCV}$ population. Anecdotal reports of symptom relief by the use of HT in patients with HCV have been reported by clients and HT practitioners. However, scientific evidence to support these claims was not available. It is important that nursing studies examine $\mathrm{HT}$ as an intervention that maybe useful in the reduction of fatigue in patients with HCV. Research to provide support for evidenced-based support for HT practice is in its infancy. The purpose of this study was to pilot test a protocol to provide preliminary data for guiding a larger study of HT and fatigue in the HCV population. 


\section{Chapter 2}

\section{Review of the Literature}

This chapter presents a review of Rogers' science of unitary human beings, the theoretical framework guiding this study, as well as four bodies of literature related to Healing Touch, Hepatitis $\mathrm{C}$ virus, treatment, and fatigue and Hepatitis $\mathrm{C}$ virus. Rogers' Science of Unitary Human Beings

Rogers' (1970) science of unitary human beings was used to provide the conceptual framework for this study. Rogers introduced a worldview of the universe as an open system and a unitary view of human beings. Within the Rogerian model the longestablished importance of adaptation, homeostasis, and equilibrium was replaced by emphasis on mutual process, homeodynamics, and unitary unfolding (Rogers, 1992).

Assumptions underlying Rogers' science of unitary human beings are that (1) a human is a unified energy field which is more than the sum of its parts, (2) a human and their environment are continuously, simultaneously and mutually exchanging energy with each other, (3) this process evolves irreversibly and unidirectionality, (4) the identity of the human energy field (person) is identified through pattern and frequency, and (5) a human is complex and characterized by his capacity for abstraction and imagery, language and thought, sensation and emotion (Rogers, 1970).

Energy fields, openness, pattern, and pandimensionality are the major concepts fundamental to this framework (Rogers, 1992). An energy field is the fundamental unit of 
a human being and the environment. Each human energy field is unique and is in continuous motion and simultaneous interaction with the environmental field. The environmental energy field is defined as all that is external to a given human energy field (Rogers, 1970). Human and environmental energy fields are inseparable, irreducible and coexist integrally with each other as an irreducible whole. Imaginary boundaries may be imposed for the purpose of study.

Openness describes human and environmental energy fields as having no boundaries and both as always being open for mutual exchange. The constant dynamic interchange of energy and materials between a human being and their environment in which one affects and is affected by the other characterizes this openness (Rogers, 1970).

Pattern is the distinguishing character of an energy field. Pattern is an abstraction which is not observable but which gives identity to the field as a whole and is perceived as a single wave. Manifestations of field patterning emerge from the mutual change process of person-environment interaction and can be observed as distinguishing characteristics (Rogers, 1980). The underlying pattern of a human being is manifested as the physical body and behaviors of that person (Malinski, 1986). Rogers discards causal and linear thinking and identifies this change as continuously innovative, unpredictable and increasingly diverse.

Rogers uses pandimensionality to distinguish a nonlinear way of seeing all reality as unitary without time or space attributes (Rogers, 1992). Within this view the human and the environment are described as negentropic energy fields engaged in a continuous mutual process that is not limited by restraints of space and time. 
Based on these assumptions, the model postulates three principles of homeodynamics; resonancy, helicy, and integrality, to explain the nature and direction of change in human-environmental field patterning. Change proceeds by the continuous repatterning of the field. The principal of resonancy is defined as the continuous change from lower to higher frequency wave patterns in human and environmental fields. The nature and direction of the change described as continuous, innovative, and unpredictable with increasing diversity of human-environmental field patterns is specified by the principle of helicy. The principle of integrality describes the nature and predicts the direction of change in the continuous mutual human and environmental field processes (Rogers, 1992).

Within Rogers' framework, a HT practitioner and the individual receiving HT was viewed as energetic beings involved in a mutual energy exchange. The energy used in HT interactions was conceptualized as a higher frequency environmental field that is integral and resonates with the human energy field. The practitioner aligns himself with a Higher Power or Universal Energy Field and does not use his own energy for healing (Hover-Kramer, 2001). Although the underlying mechanism of the effects of HT is not yet known, during a HT treatment the practitioner uses purposive patterning of hand movements with focused intention while interacting with the client to facilitate selfhealing. It is believed HT effects a change through repatterning and restoring of the energy flow and balance in the human energy field.

Health and illness are viewed as manifestations of patterns of energy. Healing is viewed as a movement towards harmony of the energy field. The belief is that in health the pattern is balanced and flowing, while in illness it is blocked, disrupted, depleted, and 
unbalanced. Rogers proposed that nursing interventions, such as HT that effect the energy field promote human-environmental patterning in the direction of shorter wave and higher frequency patterns (Rogers, 1970).

A person can experience fatigue as an energy field disturbance. In this study, the intensity of fatigue was viewed as a manifestation of the process of patterning. Self-report of reduced fatigue indicated change towards shorter wave, higher frequency, and more diverse patterning. The patients knowing involvement in the study included their expectation that they could be receiving HT and that they could potentially experience a decrease in fatigue related to this experience.

\section{Healing Touch}

Healing Touch is an energy-based therapeutic approach to healing that uses a variety of techniques to influence the energy system and promote health. In 1989, Mentgen developed the HT curriculum from her private clinical practice. The following year it grew to a certificate program that was offered in 25 locations throughout the USA and was endorsed by the American Holistic Nurses Association. The core curriculum of HT incorporates over 20 standardized, noninvasive energy-based techniques that are used to treat the human energy system by affecting physical, emotional, mental, and spiritual health of the individual (Healing Touch Notebook, 2007). Content of the five sequenced levels of classes acknowledges the work of several individuals including (Joy, 1979; Mentgen, 1993; Bailey, 1984; Brennan, 1987; and Bruyere, 1989; Scandrett-Hibdon, 1996; Hover-Kramer, 2002). National certification as a practitioner is available through either the Healing Touch Program or Healing Touch International after completion of the program of study and a one-year mentorship. The HT program is endorsed by the 
American Holistic Nurses Association and is open to all professional and lay individuals with a desire to help others. Healing Touch has been taught to over $\mathbf{8 5 , 0 0 0}$ individuals in the US and internationally and has been incorporated within the health care delivery system in hospitals, clinics and private practices.

A central belief of the HT program is that the ability to heal or restore wholeness is an innate capacity of all living things. Although all healing is understood as selfhealing, it is believed that a practitioner can connect with a client to enhance this process by using HT. All the techniques of the HT program are considered to belong to the domain of energy therapies. Healing Touch techniques vary from full body to localized interventions that have specific uses. These interventions are performed with or without actually touching the physical body and usually take from 10 to 30 minutes to complete. The goal of HT is restoration of wholeness in the energy system which including prevention of illness, promotion of wellness, and re-establishment and maintenance of health. Healing Touch is based on a holistic view of health and illness and taught as a nursing intervention and adjunct to standard health care practices (Healing Touch Notebook, 2007).

Healing Touch as an area of clinical practice is growing rapidly, however studies to support the efficacy of this treatment are limited. As of December 2008, the HT International Research survey reported almost 100 completed works, which included published research studies, doctoral dissertations, masters' theses, pilot studies, surveys, quality assurance reports, questionnaires, performance evaluations, satisfaction surveys, case studies, tools, and projects (HT International, 2008). Some of these works were not published in journals, not easily accessible, had incomplete data or design information 
and/or lacked methodological rigor, all of which compromised the accuracy and quality of the results. Only published studies and dissertations are included in this review of the literature.

\section{Healing Touch Studies}

Using an experimental design, Silva (1995) studied the effects of HT on the recovery level of post-anesthesia patients after an abdominal hysterectomy. The theory of living organisms as multidimensional fields of subtle electromagnetic energy was used as the framework. Sixty preoperative patients between 35-65 years old, scheduled for an abdominal hysterectomy in one of two private hospitals were randomly assigned to one of three groups. Group one received 20 minutes of HT, group two received a 20-minute back massage, and group three received standard treatment. All the interventions were provided by the investigator on postoperative day 1,2 , and 3 . Baseline measures were obtained prior to initiation of the interventions. Repeated measures of the variables, recovery index level and relaxation response were collected for each group of 20 subjects. The recovery index was developed for the study and included the total sum of assessments of pulmonary, gastrointestinal, urinary, and motor activity. Relaxation response measures were blood pressure, pulse, and respiratory rate. Daily records were kept of the amount of self-administered narcotic analgesia and the number of medications and treatments used to stimulate bowel function. Statistically significant results were reported in pulmonary, gastrointestinal, and motor activity for subjects who received HT compared to the other groups. Systolic and diastolic blood pressures and pulse rates were reported to be statistically significantly lower for the HT group. Less narcotic use and less bowel treatments were reported by the HT group. Theses findings supported 
integrating HT into standard care, once a day for the first forty-eight hours of postoperative care supported recovery of patients following an abdominal hysterectomy.

The strength of this study included random assignment of subjects to groups and several statistically significant findings. Having the researcher provide the $\mathrm{HT}$ and the massage interventions as well as collect data was a weakness in the design. Providing HT requires that the practitioner mentally release any personal attachment to the patient outcome and allow the energy repatterning to manifest in whatever way it does. It is debatable if one could remain centered, be fully present as a practitioner, while also having the dual role as the principle investigator perhaps eager for a specific outcome. Details of sample acceptance and attrition and measures were not reported.

Slater (1996) studied the safety and effects of HT on chronic non-malignant abdominal pain in hospitalized patients. Quantum physics and electromagnetic fields theory were used to frame the study. Selection criteria included abdominal surgery completed at least four weeks prior to enrollment with continuing pain associated with the operation. Twenty-eight subjects enrolled but five did not complete the interventions. Reasons for attrition were not reported. Subjects reported a wide duration of previous surgery completion dates ranging from less than one to nine years. The interventions included HT provided by an experienced practitioner, HT provided by an inexperienced provider and an interview. A total of 32 individuals were hired to provide the interventions, ten provided HT, ten interviewed subjects, and twelve provided sham treatments. A local coordinator was paid to run the study at each of the eight sites used. The treatment order was randomly assigned. Each subject acted as their own control and received three different interventions on separate days. The experienced and 
inexperienced providers used the same hand patterns of two HT techniques, the magnetic unruffling (later renamed the magnetic clearing) and wound sealing. Pain was measured using the McGill-Melzack Pain Questionnaire (MMPQ) before and after each treatment. Qualitative data of the experiences of the practitioner and subject were collected and analyzed by content analysis.

All treatments were found to be safe with no statistically significant differences in pain perception as measured by the MMPQ in either treatment group. Two puzzling findings were that several inexperienced practitioners reported physical discomfort providing the treatment and that the subjects reported experiencing more nausea, headaches, dizziness, and drowsiness after treatments given by a more experienced practitioner. In contrast, in the qualitative data analysis subjects reported less pain and more relaxation after HT given by an experienced practitioner. Suggested rationale for the lack of significant findings included that the MMPQ did not capture the dimensions of pain experienced, the wide range of pain duration and timing of providing the interventions.

Using an experimental pretest-posttest design and a psychoneuroimmunology framework Wheeler-Robins (1999) studied the effects of HT on well-being, neuroendocrine function, and immune function in individuals with HIV disease. To allow all patients to receive treatment a wait list cross over control group was used. Subjects were randomized to group. Thirty males diagnosed as HIV positive were enrolled, and 27 completed the weekly 30 -minute session of HT over four weeks. Females were excluded from the sample because of the possible impact of gender on neuroendocrine and immune functions. All five practitioners who provided the intervention had completed four levels 
of the HT curriculum. Only one HT intervention technique, the chakra connection was used in this study. Psychosocial measures included the General Well-Being Schedule, Faces Scale, Human Field Image Metaphor Scale, Brief POMS, and the Impact of Events Scale. Physiological measures included pulse measured pre and post treatment, salivary DHEA and cortisol, serum 5-HT as well as selected cytokine levels.

It was hypothesized that HT would enhance well being, increase levels of serum serotonin and DHEA, decrease levels of salivary cortisol and enhance immune function in persons with HIV disease. All hypotheses were rejected. Several explanations for these findings were that the treatment did not work, treatment length was insufficient, use of one standard prescribed HT technique was ineffective, ceiling effects of well being and immune function may have been present, the small sample size, or the use of ineffective psychoneuroimmunology measures. Small sample size was identified as the most significant limitation. Recommendations for future studies included using a larger sample size with estimation of effect sizes for a longer duration with more frequent HT interventions. Detailed documentation of sample selection, methods, procedures, and findings were a strength of this study. Although an attempt was made to pair subjects and practitioners, the dyads were not consistently paired throughout the study.

Geddes (1999) completed a phenomenological study that used heuristic inquiry to explore the experience of personal transformation of eight certified HT practitioners. Newman's theory of health as expanding consciousness was used as the framework. Data was collected by the researcher through observations as a participant in HT class and taped interviews that were later transcribed. Structural themes, core processes, individual depictions, composite depictions, and creative synthesis were identified. Analysis of the 
data revealed that each individual practitioner's data was unique. Structural themes that emerged from the data that were common to all were a sense of immediate rightness of the experience of HT, unanticipated personal implications and provision of a new lens to view one's life and circumstances through. Geddes study was the first qualitative study of HT to analyze the practitioners' transformation, it added descriptive data about the HT experience and support for Newman's theory of health as expanding consciousness.

In a pilot study, Krucoff et al. (2001) examined the effects of applying four nonpharmacological therapies to patients undergoing percutaneous coronary intervention (PCI) for unstable coronary syndromes. It was hypothesized that the four interventions would reduce anxiety, pain, and distress and enhance the efficacy of pharmacological agents. One hundred and fifty male subjects were enrolled and randomized to one of five groups that received; either standard care, stress management training, guided imagery, a HT technique (Chakra Connection), or double-blinded off-site prayer. Eighty-eight per cent of eligible individuals enrolled in the study. Of the 120 subjects in the four treatment groups, 118 (98\%) completed the study. Data collected on all subjects included post PCI ischemia; myocardial infarction, heart failure, urgent revascularization, and reports of mortality for six months post PCI. No results were statistically significant; however, a 25 to $30 \%$ reduction in adverse periprocedural outcomes in the experimental groups compared to the control group was reported. Patients in the off site prayer group had the fewest short term and long-term complications following PCI. No findings specific to the HT group were reported. Researchers suggest replication of the study with four times the number of individuals. 
Using an experimental with repeated measures design, Rexilius, Mundt, Megel and Agrawal (2002) examined the effect of HT and massage therapy on anxiety, depression, subjective caregiver burden, and fatigue experienced by caregivers of patients undergoing autologous hematopoietic stem cell transplant. Forty-four caregivers were enrolled and assigned to one of three groups. Each group was randomized to the interventions that were two 30-minute treatments per week for three weeks of either massage therapy or HT. The third group was for control and they received a 10-minute supportive visit from one of the researchers. All caregivers completed the Beck Anxiety Inventory, the Center for Epidemiologic Studies Depression Scale, the Subjective Burden Scale, and the Multidimensional Fatigue Inventory-20 before and after each treatment. Eight subjects did not complete the study, three reported being too busy providing care, two left after being informed the patient did not qualify for transplant, one no longer was the primary caregiver, one spouse died, and one did not complete the post-tests. Initially there were 15 subjects in the control and HT group and 14 in the massage group. After attrition $(n=8)$ there were 10 subjects in the HT group and 13 subjects in both the massage and the control group. More female than male subjects were in both intervention groups.

No results were statistically significant among the Healing Touch group. Caregivers in the massage therapy group had declines in anxiety and depression, as well as general, motivational, and emotional fatigue. In the HT group, scores decreased for anxiety and depression and increased for fatigue and subjective caregiver burden. During the study, the transplant unit converted from a hospital inpatient program to an outpatient cooperative care unit, which increased the caregiver responsibilities considerably and 
may have affected the findings. In the HT group all ten caregivers were in the outpatient cooperative care setting, their report of increases in fatigue and subject burden likely resulted from this change in the model of care.

Recommendations for future studies included using a large sample in a consistent setting, considering caregiver gender, and using different interventions. Future researchers were advised to use different individuals to provide the interventions from those who collect the data. Although power analysis and effect size were determined, attrition led to a smaller than recommended sample size which may have contributed to the lack of statistically significant findings.

Taylor (2001) investigated the effect of HT on coping ability, self-esteem and general health in nursing students using a mixed pretest posttest quantitative and qualitative design. Fifty-one participants were recruited from a four-year undergraduate nursing program and received one HT treatment each week for four weeks. Practitioners and students were not consistently matched. Findings revealed no effect of HT on the coping ability, self-esteem and general health of the first year students, however slight effects were reported for the third year students. Qualitative data supported that HT was a positive experience for all participants. The researcher suggested that the questionnaire used may have been ineffective in measuring the effects of HT and that objective measures may have been inadequate for exploring subjective experiences of HT.

Wilkinson et al. (2002) studied 22 subjects to determine the clinical effectiveness of HT on health enhancement and if the level of training of the practitioner moderated treatment effectiveness. A quasi-experimental mixed-method repeated measures design was used. The interventions were HT, HT plus music and guided imagery and no 
treatment. Standardized tools were not used. Measures included IgA in saliva, stress level self-reports, clients' perception of health enhancement and qualitative questionnaires of individual effects. Immune globulin A antibodies ( $\operatorname{Ig} \mathrm{A})$ protect body surfaces that are exposed to the outside from foreign organisms and substances can be found in saliva and can be monitored as a an indicator of immune functioning. Sample size was determined through a power analysis and predicted a medium effect size with 25 subjects; however, the sample consisted of only 19 females and 3 males. The nine female practitioners giving the interventions had from 3-11 years of experience providing HT. The same practitioner-client dyads participated in all conditions in an effort to assure consistency. Practitioners were not restricted to one particular HT technique but were encouraged to utilize normal HT practice. Results revealed statistically significant higher IgA levels across the HT treatments for subjects of more experienced practitioners. Although not statistically significant, subjects in both HT intervention groups reported a reduction in stress level. Qualitative data revealed themes of relaxation, connection, and enhanced awareness. Fifty-five per cent $(n=6)$ of the clients who reported experiencing pain at the start of the study reported pain relief after HT. The researchers suggest the data support the clinical effectiveness of HT in health enhancement, lowering stress perceptions, and relieving pain. A study with a larger sample size was recommended to further assess the effectiveness of the treatment related to the level of training of the practitioner.

The researchers acknowledged that the background education and training of practitioners in other healing modalities might have influenced the results. Sample selection procedures allowed some practitioner and subject dyads to know each other prior to the study, which may have affected the findings of relaxation and connection. 
Post-White et al. (2003) tested the use of therapeutic massage, and HT, compared to presence or standard care to inhibit relaxation or reduce symptoms in patients with cancer receiving chemotherapy. The design was a randomized, prospective two-period crossover intervention study. After a $29 \%$ attrition rate, 164 subjects completed the study allowing detection of a small to medium effect size. Fifty-six subjects received HT, 63 received massage, and 45 were in the presence group. Subjects received 4-weekly, 45minute interventions and 4-weekly sessions of standard care. The condition sequence was randomized. Measures before and after each intervention included: heart rate, respiratory rate, blood pressure, and pain and nausea on a scale of 0-10. The Brief Pain Index, Brief Nausea Index, and fatigue, anxiety, and mood by the Profile of Mood States were measured before the first and fourth intervention. Subjects completed daily diaries of anti-emetic and analgesia use that were collected weekly. The same practitioner/subject dyad was maintained in most cases. Results indicated that blood pressure, heart rate, respiratory rate, and pain were lowered by healing touch and massage therapy. Anxiety was lowered by massage therapy and fatigue and total mood disturbance were lowered by HT. A lower amount of non steroidal medication was used in week 4 with massage therapy. There were no effects on nausea. Presence did not differ from standard care on any measure except heart rate and respiratory rate. It was noted that massage therapy and HT were more effective than presence or standard care on pain, mood disturbance, and fatigue in patients with cancer during chemotherapy treatment.

One of the strengths of this study was the long duration of 33 months for the enrollment of participants. At the end of the study, a total of 164 subjects had completed all 8 sessions. An additional strength was that descriptions of the cross over study design, 
methodology and statistical analysis and findings were explained in thorough detail. Currently the findings from this study provided the largest base for an outcome study of HT.

Loveland Cook, Guerrerio, and Slater (2004) conducted a two arm experimental study to test the efficacy of HT on quality of life during radiation treatment for 62 women diagnosed with breast or gynecological cancer. Enrollment of subjects lasted for 24 months. Initially 78 subjects consented and were randomized to either HT $(n=44)$ or mock treatment $(n=34)$. Depending on group placement each subject received either six HT or six mock treatment interventions. Mock treatment was a visit from the mock provider. The researchers were blinded to the group assignment and did not provide any of the interventions. Sixteen subjects ( $21 \%$ ) failed to complete the study, ten in the HT group and six in the mock treatment group. In the final analysis, 34 subjects were in the HT treatment group and 28 were in the mock treatment group. The timing of the interventions was once within the first third of radiation treatments, followed by four sessions once a week and one final session four weeks later. Measures included a sociodemographic survey; a 4-item questionnaire of attitudes toward HT and the SF-36 to measure health related quality of life. The findings reported baseline sociodemographic characteristics and attitudes toward HT were similar for both groups. In all nine domains of the SF-36, the HT group scored higher than the mock treatment group with statistically significant differences reported for 3 subscales, vitality, pain, and physical functioning. Significant within-group changes in emotional role functioning, mental health and health transition were found for the HT group. For the mock treatment group statistically different change scores were found in physical role functioning and health transition. 
Wardell (2005) used a mixed design pilot study to investigate the use of HT in effecting the perception of chronic neuropathic pain in 29 patients with spinal cord injuries from a Veteran's Affairs Medical Center. The participant received either HT or controlled progressive muscle relaxation during 6 in-home visits. Quantitative analysis was conducted with pre and post data measured by the Profile of Moods States (POMS), the Brief Pain Inventory, the Diener Satisfaction with Life Scale and the Center for Epidemiological Depression scale and a VAS. Qualitative analysis of the focus group and interviews of the participants and providers perceptions of the experience were completed. The qualitative data revealed one participant had an initial decrease in pain that later returned, one reported overall relaxation with no change in symptoms, and 2 of the five HT participants reported significant pain reduction. Three subjects reported no perceived change. Participants in the control group reported overall relaxation. The practitioner's responses varied from positive with significant pain symptom improvement to a hindrance created by the participants' hostile attitude. Quantitative data was significantly different for the Composite of Interference on the Brief Pain Inventory $(t=2.71, p=.035)$ and there was a decreasing trend of fatigue subscale in the POMS. The Diener Satisfaction with Life Scale measured an increase in satisfaction in the HT group. Depression decreased in the HT group but there was not a significant change between groups on this variable. Weekly VAS scores of pain and coping showed an initial decrease that was not sustained before the next treatment. The researcher recommended a future larger study with three arms as the relaxation intervention may have contributed to pain reduction. Changing the location for the interventions from patients' homes as well as measuring patients and practitioners expectations were suggested for future studies. 
Van Aken (2004) completed a grounded theory with a case study perspective design to explore the process of HT in individuals emerging from moderate depression. The sample of 15 self-referred participants received five weekly sessions of a variety of HT techniques by the PI after verification of their depression by the Beck Depression Inventory. The Healing Energy and Life Through Holism (HEALTH) tool, an unstructured interview at week 6 and a second Beck Depression Inventory were the measures used for data collection. Structural themes that emerged from the data revealed a four stage experiential process of the HT sessions in individuals with moderate depression. It was reported that stage one provided the opportunity to experience understanding, safety, trust, and positive anticipation by belief in the practitioner, self and future self. In Stage 2 the experiences of body awareness, spiritual awareness, mental harmony and emotional harmony allowed for integration of self in the reconnection process. The experience of feeling heartened, healing, acceptance and having clarity were accessed by connection with inner strengths and resources in the third stage. The fourth stage was the process of being motivated and embracing change in which the individual engages with life and experiences of the ability for involvement, looking outside, becoming motivated and embracing change. This first grounded theory of HT and individuals with moderate depression added descriptive data for understanding the process of Healing Touch and was suggested for use by practitioners when working with individuals with moderate depression.

Wang and Hermann (2006) conducted a pilot study over eight weeks using a nonrandomized quasi-experimental design to examine the effects of HT on agitation in male patients with dementia. The Cohen-Mansfield Agitation Inventory (CMAI) measure 
was given by a licensed vocational nurse (LVN) to all 14 subjects daily for two consecutive weeks prior to any intervention. The sample consisted of all residents on one dementia special care unit. The six highest scorers on the CMAI were chosen as the patients in the treatment group and the remaining eight subjects were the control. The HT techniques used were the modified mind clearing and unruffling (since renamed handsin-motion). HT was provided for 10 minutes once a day for 4 consecutive weeks and the measure was given once a week. The control group received normal care. During the final two weeks of the study, the data was collected on the overall group to provide more data for the non-treatment scores. Data analysis revealed that the experimental group mean had a significant decrease in agitation compared to the control group during the same weeks. The data collector remained blinded throughout the study. The practitioner providing the HT reported the participants stated the intervention was enjoyable. Having male patients and a female HT practitioner was suggested as a possible confounding variable. The investigator recommended a larger and longer duration study with randomization and different measures.

In this study, the results were reported as highly significant. This claim should be viewed with caution considering the small sample size and non-randomization of the subjects to treatment.

Dowd, Kolbaca, Steiner, and Fishinpaur (2007) compared three interventions: HT, coaching, and a combination of the two compared to a waitlist for increasing comfort and reducing stress in young college students. Using a 4-group experimental design, young volunteer students from two sites at one university campus who self-identified as having multiple stressors and associated symptoms were randomized to 1 of 4 groups. 
The dyad of the initial nurse, who provided coaching and HT and the student was consistent for the duration of the study. The mind clearing created by Rev. Rudy Noel was the HT technique provided. The waitlist group completed online questionnaires at preset schedules. Comfort was measured using two measures, a 0-10 rating scale for comfort and the Healing Touch Comfort Questionnaire (HTCQ). Stress was also measured with two scales: a 0-10 rating scale for stress and the stress test measure. Data was collected at baseline, before the first intervention, immediately before the third intervention and after the treatment protocol was completed. Once-a-week interventions were provided over 3 consecutive weeks. Eight data sets were lost to attrition. The sample of 52 subjects were similar on stress and control at baseline and consisted of the following groups: HT 12 subjects, coaching 14 subjects, combination 13 subjects and waitlist 13 subjects. Results were significant indicating that comfort and stress had better immediate results when treated with HT, while coaching had a longer duration of effects on both. Suggestions for future research included using a 25 years and older population, randomization of subjects to group after baseline measures are completed, revision of the HT protocol to include assessment of student's symptoms related to stress and allowing a longer period to measure the treatment outcomes. This study was well designed and included clear and carefully described results with detailed statistical analyses.

In summary, the available HT literature informed the design of the proposed study. It includes studies using a variety of samples, designs, theories, measures and HT techniques with resulting mixed findings. Statistically significant findings supported the use of HT to decrease blood pressure and pulse and accelerate recovery in patients after abdominal hysterectomy (Silva, 1995). Wilkinson (2002) found statistically significant 
findings for HT being more effective when provided by a more experienced practitioner, which conflicts with results reported by Slater (1996). Statistically significant findings supported the use of HT for agitation in patients with dementia in a study by Wang and Hermann (2006). Dowd et al. (2007) found statistically significant findings for the immediate effects of HT on comfort and stress in younger aged college students.

Three studies using different patient samples and different standardized tools have measured HT and fatigue. Fatigue measured by the Multidimensional Fatigue Inventory20, increased after HT treatment in the study by Rexilius (2002). Fatigue measured by the SF-36 decreased in the study by Post-White et al. (2003) and also when measured by a subscale of the POMS by Wardell (2005). These beginning studies with conflicting results identify the need for further investigation to evaluate the effectiveness of HT to treat fatigue.

Use of a consistent theoretical framework and building on the findings of preceding studies would strengthen the HT research. The studies reviewed identified the number of subjects enrolled; however, only the studies by Rexilius et al. (2002), Loveland Cook et al. (2004), Post-White et al. (2003) and Dowd et al. (2007) provided details of subject attrition and used power analysis for determining sample size. WheelerRobins (1999), Krucoff et al. (2001), Wang and Hermann (2006) and Dowd et al. (2007) specified, described, and included a rationale for the specific HT technique used.

In HT studies, a common problem is underreporting of data including sampling procedures, data collection schedules and procedures, and instruments. In addition, the HT techniques utilized and the theoretical or conceptual bases for the study often are not reported. The small number of studies, the insufficient reporting of data, the variety of 
designs and resulting mixed findings, highlight the need for further research to investigate the efficacy and use of HT.

Hepatitis C Virus (HCV)

Hepatitis $\mathrm{C}$ virus, the most common chronic blood borne viral infection in the United States was identified in 1989. It has reached epidemic proportions with an estimated incidence of 170 million individuals worldwide and 3.2 million in the US. Infection with $\mathrm{HCV}$ is the number one reason for liver transplant, a complex and costly treatment. Treatment for HCV has only recently become available and testing for the virus has only been possible since 1992 . A HCV vaccine is not yet available. Seventy to $85 \%$ of $\mathrm{HCV}$ infected persons are asymptomatic for 20 years or more after contracting the virus. These individuals often remain unaware of their diagnosis and therefore do not seek early treatment and may unknowingly transmit the HCV to others. Shared needles used by IV drug users and multiple sexual contacts contribute to the silent spread of HCV (CDC, 2008).

Risk factors associated with HCV incidence include being male, aged 30-49 years, or African American. The two most common causes of HCV transmission are receiving a blood transfusion prior to 1994 and illegal drug injections. In the US the primary risk factor, IV drug use is responsible for $69 \%$ of $\mathrm{HCV}$ transmission (Strader, Wright, Thomas, \& Seeff, 2004).

Sixty to seventy percent of patients with acute HCV may be asymptomatic or develop only mild symptoms 4 to 12 weeks after exposure to the virus. Seroconversion occurs between 8 and 24 weeks. In 15-25\% of patients with acute HCV, the disease will resolve without any sequela. The remaining patients experience a slow asymptomatic 
progression over 20 to 40 years resulting in chronic $\mathrm{HCV}$ with liver damage leading to possible cirrhosis and an increased risk for liver cancer (CDC, 2009).

Chronic Hepatitis C Virus and Pegylated Interferon and Ribavirin Treatment

Eradication of the virus, the primary goal of $\mathrm{HCV}$ treatment can currently be achieved in over $50 \%$ of treated patients (Terrill, 2004). The American Association for the Study of Liver Disease (AASLD) by Strader, Wright, Thomas and Seeff, (2004) standardized the recommendation for treating HCV. Studies support the standard combination treatment of pegylated interferon and ribavirin as the current optimal therapy for chronic HCV because it provided the best chance for a cure (Bacon, 2004; Frederick-Rust, Zuezem, \& Sarrazin, 2007; Kjaergard, 2002; Rasenack et al., 2003). Recommendations for continuation of this combination treatment were provided in the current Management of Chronic Hepatitis C Canadian Consensus Guidelines (Sherman et al., 2007).

Interferons are a family of naturally occurring small protein molecules that are produced and secreted by cells in response to viral infections. Interferons function by modifying the response of the body's immune system to help fight infections. Pegylated interferons (PEG) are a slow release longer lasting form of alpha interferon in which the interferon is attached to a molecule of polyethylene glycol. Patients using PEG interferon required only one self-injection a week. Interferon combined with ribavirin provided a substantially increased response rate of $40-50 \%$ when compared to the response rate of 15-25\% for interferon alone (Fried, Shiffman, Reddy, Smith, Miarinos et al, 2002). Increased benefits of combined use of interferon and ribavirin were also supported in a study by Hadziyannis, Sette, Morgan, Balan, Diago, Marcellin, et al. (2004). 
Ribavirin is an antiviral synthetic nucleoside analog that is not effective against HCV unless combined with interferon. Ribavirin was taken orally twice a day in the morning and evening. An additional benefit of ribaviran was a significantly result of decreased the relapse rate when compared to the use of interferon alone (Fried et al., 2002).

Table 1

Contraindications for Starting Combination Interferon and Ribavirin Treatment

\begin{tabular}{ll}
\hline \multicolumn{1}{c}{ Contraindication } & \multicolumn{1}{c}{ Reason } \\
\hline Alcohol consumption & Poorly controlled hypertension \\
Using illicit drugs & Poorly controlled diabetes \\
Unwilling to comply with contraception method & Anemia, neutropenia or \\
\multicolumn{1}{c}{ during and 6 months post treatment } & thrombocytopenia \\
Hemoglobinopathies & Active autoimmune disease \\
Pregnant or lactating mothers & Liver decompensation \\
Poorly controlled psychiatric illness & INR> 1.3 \\
Depression & Poorly controlled seizure disorder \\
Severe or debilitating medical conditions & Chronic renal failure \\
Severe cerebrovascular disease & Active autoimmune disease \\
Poorly controlled congestive heart failure, or & Liver decompensation \\
\hline arrhythmia & \\
\hline
\end{tabular}

Two drug companies supplied this antiviral combination therapy, Roche as PEG interferon alfa 2a (Pegasys) + Ribaviran (Copegus) and Schering as Peginterferon alfa-2b 
and Ribavirin (Pegetron). A comparison of two large studies of each drug concluded that either drug can effectively treat chronic HCV $50 \%$ of the time (Bacon, 2004). Subjects included in the study reported here used antiviral combination therapy made by both companies.

Table 2

Drugs Used in the Treatment of Chronic Hepatitis C Virus

\begin{tabular}{ll}
\hline Generic (Trade Name) & Recommended Dose \\
\hline Peginterferon alfa-2a & 180 micrograms SQ once weekly \\
$(40 \mathrm{kD})$ (Pegasys) & Regardless of weight \\
Peginterferon alfa-2b & 1.5 micrograms/kg SQ once weekly \\
$(12 \mathrm{kD})$ (Peg-Intron) & \\
Ribavirin (Rebetol, Copegus) $800-1200 \mathrm{mg}$ orally daily (in two divided doses), dose \\
depends on infection, genotype, and patient weight.
\end{tabular}

The strongest predictor of response rate to a combination of interferon and ribavirin treatment varies according to the six different genotypes of $\mathrm{HCV}$ that have been identified. Once a patient's HCV genotype is identified, it does not change over the course of the infection. In the US $70 \%$ of $\mathrm{HCV}$ infections are genotype one. These individuals have a substantially lower response rate to combination treatment than those with genotype two or three. Patients with genotype two or three are almost three times as likely to respond to combination interferon and ribavirin therapy compared to those with genotype one. In patients with genotype 1 a lower viral load is predictive of a higher sustained response rate $(73 \%)$ when compared with genotype one patients with a higher 
viral load and lower response rate (37\%). The recommended duration of therapy for patients with genotype one was 48 weeks, while patients with genotype two or three required only 24 weeks of treatment (Strader, Wright, Thomas, \& Seeff, 2004; Ward, Kugelmas, \& Libsch, 2004, Zic 2005).

Recognition and management of the side effects of combination treatment with interferon and ribavirin is essential for effective patient care. Although fatigue was the most commonly reported side effect, depression, hallucinations and suicidal ideation induced by interferon have been a significant reason for early termination of treatment (Crone, Gabriel, \& Wise, 2004; Zichmond et al, 2006 ). These serious side effects can occur in patients treated with interferon without any previous history of psychiatric problems. Neutropenia associated with interferon use and anemia associated with ribavirin use can result in dose reduction or discontinuation of treatment (Stader Wright, Thomas, \& Seeff, 2004).

McHutchison (2002) reported that if patients remained adherent to the recommended dose and duration of the standardized combined therapy, response rates of up to $50 \%$ in patients with genotype one and from $56-80 \%$ in patients with genotypes two or three were sustained. This was a significant finding indicating that HCV could be eradicated in a higher number of patients if they adhered to the treatment regimen with interferon and ribavirin. Studies to support recommendations for combination treatment of patients in genotypes four, five, and six were not yet available. Physicians were advised to make treatment decisions for this group of patients on a case by case basis (Strader, Wright, Thomas, \& Seeff, 2004). 
Fatigue and HCV

A clear definition of fatigue experienced in patients with chronic $\mathrm{HCV}$ was not found. One exploratory study suggested that fatigue experienced in HCV patients is a major problem and so unique that it should be reconceptualized as lassitude to be distinguished from the fatigue that everyone experiences on a daily basis. Dysfunctional lassitude was defined as weariness without exertion, lack of recovery of energy with rest, rapid and dramatic weariness, and interference with vocational and social roles (Vallis, Peltekian, \& Hirsh, 2003). Numerous studies of symptoms occurring in patients with chronic HCV supported fatigue as the most frequently occurring and significant side effect (Bacon, 2004; Glacken et al., 2003; Hassoun, Willems, Deslauriers, Nguyen, \& Huet, 2002; Huber, 2001; Kallman, ONeill, Larive, Bopari, Calabrese, Younossi, 2007; McDonald, Jayasuriya, Bindley, Gonsalvez, \& Gluseska, 2002; Poynard et al., 2002; Schaefer, 2004; Vallis, 2001). Hirsch et al. (2001) reported that $69 \%$ of all patients with $\mathrm{HCV}$ at the same clinical site where this present study was completed experienced fatigue.

Glacken et al. (2003) identified fatigue as being multidimensional. Using grounded theory and interviews from 28 patients with chronic $\mathrm{HCV}$, descriptions of two types of fatigue emerged as chronic or permanent and idiopathic or transient. Both types had physical, cognitive, and affective dimensions that were experienced at differing intensities by each individual. Severity of the fatigue was considered a combination of intensity, duration, and frequency.

Several studies reported no relationship between fatigue and the severity of the disease process. Barkhuizen et al. (1999) found fatigue reported by $56 \%$ of 239 patients 
was unrelated to severity of $\mathrm{HCV}$, route of infection or interferon treatment. Sixty-seven percent $(n=92)$ of patients with chronic HCV interviewed by Hassoun, Willems, Deslauriers, Nguyen, \& Huet (2002) reported the presence of frequent and disabling fatigue that was unrelated to the severity of disease or the viral load. In a study of 115 patients with HCV, McDonald, Jayasuriya, Bindley, Gonsalvez, \& Gluseska (2002) reported a strong correlation between fatigue and depression but not between fatigue and disease severity. Poynard, Cacoub, Ratziu, Myers, Dezailles, et al. (2002) noted that in a study of patients with HCV $(n=1,614)$ fatigue was reported by $53 \%$, however it was only described as severe and impairing activity in $17 \%$ of subjects. There was no significant association between fatigue and viral load, genotype, alcohol consumption, abnormal thyroid function, or cryoglobulinemia, (an abnormal protein associated with HCV infection).

The efficacy of interferon and ribavirin treatment has been established as effective in the HCV population, however; the resulting fatigue is a prevalent and severe side effect (Ward, Kugelmas, \& Libsch, 2004). Reports of patients experiencing fatigue in the $\mathrm{HCV}$ interferon treated population range from $46 \%$ to $92 \%$ of patients receiving combination drug therapy (Arase, 2006; Davis, Beck, Farrell, \& Poynard, 1998; Zucker, 2001). Fatigue severity causes some patients to discontinue the necessary 24 to 48 weeks of medical treatment (Arase, 2006; Fried, 2002; McHutchison, 2002).

In a study using a pretest-posttest design Zucker (2001) recruited 20 patients with $\mathrm{HCV}$ who were starting combination therapy to pilot test measures of fatigue and quality of life (QOL) and the effect of an exercise intervention. Subjects were randomized to either a group receiving exercise or one receiving standard care. Subjects in the exercise 
group followed a self-paced walking program and attended a support group. Support phone calls from a nurse were provided for both groups. Measures included the Schwartz (Cancer) Fatigue Scale and the Hepatitis QOL Scale. In addition, a record of completion of the combination therapy was recorded. Pre and post-test measures of fatigue, QOL, and walking distance were taken on all subjects. The walking intervention resulted in a small to medium effect relative to reduction in fatigue, completion of combination therapy, increased QOL, and walking distance. In addition, the HQOL subscales for hepatitis specific limitations and distress were increased in the exercise group and relatively unchanged in the control group. Zucker suggested that fatigue in patients with $\mathrm{HCV}$ receiving combination therapy was rarely a single complaint, but was more likely to appear clustered with other symptoms. In addition, Zucker reported that assessment of clusters of symptoms associated with fatigue and patients with HCV have not been reported in the literature.

Carlson et al. (2004) studied fatigue and disordered sleep in 59 subjects with chronic HCV. The measures Fatigue Severity Scale (FFS) and the Pittsburgh Sleep Quality Index (PSQI) were completed by all subjects. There were no significant differences in gender on either scale. Fatigue and poor sleep quality $(r=0.58, p<0.01)$, were significantly correlated.

Summary

Identifying and treating the underlying cause of a distressing symptom is the most effective approach to symptom management; however, often no source for interferoninduced fatigue experienced by chronic HCV patients can be identified. Little is known about the optimal management of fatigue in this population and few interventions have 
been tested formally. Lack of consistent use of assessment tools and the fact that fatigue fluctuates unpredictably over the disease process add to the difficulty of treatment management. Studies investigating fatigue and hepatitis have been completed in the general hepatitis population; however, there are limited available studies of management of fatigue in adults with chronic HCV while receiving combination interferon treatment (Arase, 2006; Benson, Birkel, Caldwell, Stafford-Fox, \& Casarico, 2000; Birkel, Caldwell, Stafford-Fox, Casarico, \& Benson, 2000; Fried, 2002; Piche et al. 2005; Zucker, 2001). In the majority of these studies dose reduction is the recommended treatment for interferon-induced fatigue. One pilot study tested exercise as an effective intervention (Zucker, 2001). Other interventions recommended for fatigue in patients with HCV included the treatment of anemia and depression, exercise, administering interferon injections at night, sleep, energy conservation, stress reduction and pharmacological therapy (Huber, 2001). Based upon this review of the literature and need for research to investigate the use of HT for fatigue in patients with HCV, the purpose of this research was to pilot test a protocol using HT to relieve fatigue in patients with HCV who were being treated with combination interferon and ribavirin. 


\section{Chapter 3}

\section{Methodology}

This chapter describes the research methodology used to test a protocol utilizing a Healing Touch intervention to relieve fatigue experienced by persons with chronic $\mathrm{HCV}$ who were receiving a combination of PEG interferon and ribavirin treatment. The research design, subject and setting selection, procedures, measures and data analytic strategies will be presented.

\section{Research Design}

A pretest-posttest experimental repeated measures design was selected for this study. Subjects were randomized, a process based only on chance, to either the experimental or control group.

\section{Table 3}

\section{Experimental Randomized Controlled Design with Repeated Measures}

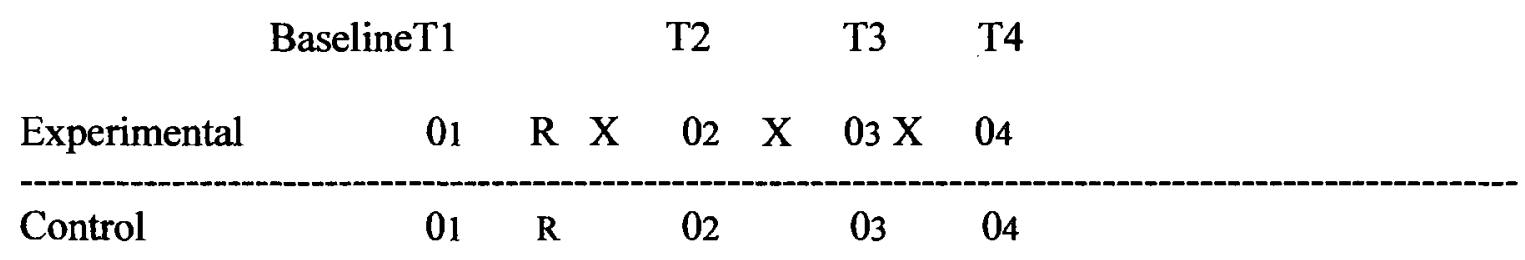

Subjects who were randomized to the experimental group received a weekly HT intervention over a consecutive 3-week period. Those in the control group received a mock touch (MT) intervention at the same time intervals. Measures of the dependent 
variable, fatigue using the Schwartz Cancer Fatigue Scale (SCFS) were obtained prior to randomization and at three other repeated intervals for subjects in both groups. Using the Fatigue Impact Scale (FIS) fatigue was also measured at baseline and again at week 4 after completion of all interventions for both groups.

Random assignment ensures that groups are as equivalent as possible prior to receiving the intervention. Therefore, outcome differences that are observed in the two groups at the completion of the study are likely from the treatment. Subjects were assigned to either group by use of a computer-generated table of random numbers. The control group was used to measure the dependent variable, fatigue in which the treatment had not been applied. Throughout the data collection procedure efforts were made to ensure similar study conditions for both groups, except for the application of the independent variable, the HT intervention.

\section{Data Collection}

Sample

The sampling frame included all patients with a diagnosis of chronic HCV being treated with a combination therapy of PEG interferon and ribavirin in a hepatology outpatient clinic in a large teaching multidisciplinary health care facility in eastern Canada. From the total provincial population of 950,000 individuals, there were $4,000-$ 5,000 infected with HCV. Every year at least 50 to 60 patients meeting these criteria were treated at this clinic. A sample size of 30 subjects was selected given the pilot design and resource constraints of this study. Twelve months were required for data collection.

Subjects were recruited through a verbal invitation by one of the two nurse practitioners to all patients attending the clinic during the timeframe of the study. It was 
difficult to predict if this would allow for a sufficient number of subjects because no previous similar research was available. The refusal rate of all patients approached and the rate and reason for subject attrition were reported.

Inclusion criteria for subjects included age of at least 18 years, a confirmed diagnosis of $\mathrm{HCV}$, receiving interferon and ribavirin for 4 consecutive weeks, ability to read, speak, and write English and to complete the required paperwork, and not be receiving other energy therapies.

\section{Setting}

The study was conducted at a large health care center with a multidisciplinary hepatology outpatient clinic in eastern Canada. The clinic was open for patients 4 days a week and was located in one wing that has offices and four patient exam rooms. Both the HT and the Mock Touch (MT) interventions were completed in the same designated clinical room in a separate outpatient building located nearby that was open Monday to Friday.

Instruments

The science of unitary human beings by Rogers (1970) was the theoretical framework selected to guide this study. Using this framework an individual is viewed as surrounded and penetrated by an energetic field that is open and can exchange energy. It is theorized that such an exchange of energy may occur between a practitioner providing HT and the individual receiving it. Fatigue was the variable measured as a manifestation of the patient-environmental field patterning process. The empirical indicators used to measure fatigue were the Schwartz Cancer Fatigue Scale (SCFS) and the Fatigue Impact Scale (FIS). 
Table 4

Theoretical Construct and Empirical Linkages

Theory Concept Energy Field/Principles of Homeodynamics

Variable Measured Fatigue

Empirical Indicators Fatigue Impact Scale (FIS)

Schwartz Cancer Fatigue Scale (SCFS)

Four instruments were used for data collection: Demographic Data Form (DDF), Schwartz Cancer Fatigue Scale (SCFS), Fatigue Impact Scale (FIS), and the Centers for Epidemiologic Studies Depression Scale (CES-D). Permission for the SCFS and FIS was obtained. The CES-D is in the public domain. One aim of this pilot was to assess the level of fatigue over four weeks. Two instruments have greater potential for capturing a change in fatigue than a single measure. For this reason, the dependent variable was operationalized by two measures, the Schwartz Cancer Fatigue Scale (SCFS) and the Fatigue Impact Scale (FIS).

Demographic Data Form (DDF). The DDF was designed for this study. It is a 13item questionnaire (see Appendix A). The DDF was used to identify age, gender, race, education, employment status, genotype, time since diagnosis, months of combination treatment, other diagnoses, and current medications. Subjects were asked for permission to review their chart to identify genotypes, date of diagnosis, number of months of combination treatment, co-morbidities including HIV status, hemoglobin level, if available during the three weeks of the study, and current medications. 
Schwartz Cancer Fatigue Scale (SCFS): The SCFS is a self-report questionnaire used to measure fatigue on physical and perceptual subscales. It is a six-item, 5-point Likert scale that is scored from $1-5$. Items range from " $1=$ not at all" to " $5=$ extremely." Possible scores range from 6-30. Higher scores indicate a higher presence of symptoms of fatigue. Crohnbach's coefficient alpha for the total scale is 0.90 and is 0.88 and 0.81 for the physical and perceptual subscales, respectively in a population of patients with differing cancer diagnoses and treatments (Schwartz, 1998; Schwartz and Meek, 1999). Subjects were asked to read printed descriptors of how fatigue made them feel in the last 2-3 days and circle the number which best indicated their response. The SCFS scale was selected because it is a short self-report questionnaire that had been used successfully in a clinical outpatient setting. Although the SCFS was developed for use in persons with cancer it has been used in a small study $(n=20)$ of patients with HCV and fatigue using an exercise intervention (Zucker, 2001). In this study the internal consistency reliability was .75. The small sample size may have contributed to lower reliability in this population. Completion of the SCFS took approximately 2 minutes.

The Fatigue Impact Scale (FIS): The FIS was used to measure patients' perception of the functional limitations that fatigue had caused during the past 30 days. It is a forty-item, 5-point Likert scale that is scored from $0-4$. Items are graded by severity and range from " $0=$ no problem" to " $4=$ extreme problem" with a possible score of 0 160. Higher scores indicate higher patient perceived functional limitations caused by fatigue. The items reflect three subscales of perceived impact on cognitive, physical, and social functioning. The maximum scores for these domains are 40,40 , and 80 respectively. Internal consistency for the three subscales was .87 (Fisk et al., 1994). This 
scale was originally used to investigate chronic fatigue in patients with multiple sclerosis. In a study of patients with chronic liver disease the FIS correlated well with fatigue assessed by a visual analog scale ( Hassoun et al., 2002; Jones, 2004; Vallis et al., 2001). The FIS was selected for use in this study because it is a brief self-reported questionnaire that took approximately 10 minutes to complete.

Centers for Epidemiologic Studies Depression Scale (CES-D): Depression measure of all subjects was completed prior to randomization because a correlation between Hepatitis $\mathrm{C}$ and depression has been previously identified in the literature. (Bacon, 2004; McDonald, Jayasuriya, Bindley, Gonsalvez, \& Gluseska, 2002; Zickmund et al., 2006). The CES-D is a self-report questionnaire used to measure presence and severity of depressive symptoms in the general population rather than a psychiatric population. This tool measures the frequency and duration of depressive symptoms in the previous week. The CES-D is a 20-item, 4-point Likert scale that is scored from $0-3$. The scoring of positive items is reversed. Possible scores range from 0-60. Higher scores indicate a higher presence of depressive symptoms. It measures the domains of depressive affect, somatic symptoms, positive affect, and interpersonal relationships. Internal consistency was .85 in the general population in the original study by Radloff (1977). In a later study of patients with HCV who were being assessed for possible treatment with antiviral therapy, internal consistency was .88 (Clark, Mahoney, Clark, \& Eriksen, 2002). The CES-D was selected because it was a self-report questionnaire that had few items and concise wording. It took subjects approximately 4 minutes to complete. 


\section{Procedures}

Following approval by Institutional Review Board of the University of San Diego and the Ethics Board of the Queen Elizabeth II Health Sciences Centre the study was initiated in July 2006. As required by the facility, the initial contact person for all individuals was one of the two NPs. A formal presentation was given by the principal investigator (PI) to the two NPs to provide information about the study, procedure for recruitment of participants, screening of subjects for inclusion criteria, acquiring the consent and providing the initial baseline measures. The PI or research assistant (RA) conducted all data collection with the exception of a list of all individuals approached to join the study, several study consents, a list of reasons for subject refusal, and several of the medical chart audits, which were collected by the two nurse practitioners (NPs). At least one of the two NPs was available each day to meet with new subjects. All patients who attended the clinic during the specified time period were invited to participate and had the study explained by one of the two NPs who regularly provided care in the clinic or were referred to the PI or RA.

A list of those individuals consented or who were interested to learn more about the study was kept by the NPs for follow up by the PI or RA weekly. Persons who requested more information were contacted by the PI or RA directly by phone, the study was re-explained, and questions were answered. Once one of these individual stated the desire to be included in the study the PI or RA met with them privately at mutually agreed upon time in the clinic to complete the consent and baseline information. Patients were screened for the inclusion criteria. The purpose and goals of the study were explained, study related questions were answered and a written informed consent was 
obtained. All subjects were informed that the study was to investigate the use of an energy-based complementary therapy in patients with $\mathrm{HCV}$ who were receiving interferon and ribavirin treatment. The possibility of receiving the MT intervention was revealed to all subjects, but they were blinded to their group assignment. A copy of the signed consent was given to each subject.

After the consent was signed, the PI, RA or one of the two NPs extracted medical information from the medical chart in order to complete the Medical Chart Audit Form. It contained information about time since HCV diagnosis, genotype, current treatment, medical co-morbidities, HIV status, current hemoglobin and concurrent medications.

Next, the self-report instruments were administered in the following order: Demographic data form (DDF), Schwartz Cancer Fatigue Scale (SCFS), The Fatigue Impact Scale (FIS), and the Center for Epidemiologic Studies Depression Scale (CES-D). To reduce subject burden and ensure completion of all items the PI or RA read all questions to the subject. Subjects were taught how to complete the SCFS for future visits. Subjects were instructed to score the SCFS two days after each intervention and deliver the documentation in a sealed envelope addressed to the PI or RA at the next clinic visit. Following the completion of all baseline data subjects were randomized to the HT or MT intervention group by use of a table of random numbers (Haahr, 2006).

Appointments to receive either intervention were available five days a week and subjects chose their appointment from available time slots. Subjects were instructed all scheduled appointment changes were to be made through the PI or RA.

Using the principal investigator to collect data as well as provide the intervention was a problem reported in a previous HT intervention study (Rexilius, Mundt, Megel, \& 
Agrawal, 2002). The PI did not provide any of the HT interventions. After baseline collection, all data were gathered by the PI, RA or self-reported by the subject. The subject, the two NPs, and the clinic physician were all blinded to whether the subject received $\mathrm{HT}$ or $\mathrm{MT}$.

Three HT practitioners provided the intervention. They all had completed a minimum of three levels of the HT program and had practiced HT consistently for a minimum of 2 years. Three experienced nurses who did not have experience with giving or receiving energy therapies provided the MT intervention for the control group. The MT intervention was taught by the PI and used a consistent different hand movement from the HT intervention. An effort was made to maintain the same subject/practitioner dyad throughout the duration of the study.

The independent variable HT was operationalized using the magnetic clearing, a HT technique that was developed by Mentgen. This technique was selected because it is recommended for use to clear congested energy in the body's energy field in a systematic way (Healing Touch Notebook, 2007). This technique was completed in fifteen minutes and was done without the practitioner's hands touching the subject. The time of thirty minutes for the entire session was chosen to allow for usual practice of HT (Appendix B).

The mock treatment (MT) was developed for this study by the PI and involved hand movements beside either side of the body for fifteen minutes, which was the same length of time as the HT intervention. The MT providers were instructed to perform mathematic calculations of counting backwards by sevens and to focus their thoughts away from the subject while providing the intervention. The entire session lasted for 30 minutes to provide consistency in both groups (Appendix C). 
Subjects in both groups received the usual standard care provided at the clinic during the time of the study. The RA was a research nurse who was employed in the clinic. Prior to the beginning of the study, a meeting was held by the PI with the RA to establish consistency in explaining the study and giving the measures. All interventions for both groups were provided between the hours of 0800 and 1700 on Monday to Friday when the facility was open. Measures were taken during four consecutive weeks, to allow for the effects of the HT intervention while providing short study duration for each individual. Measures were consistent related to times and measures within and across groups (see Table 5). 
Table 5

Data Collection Procedures

\begin{tabular}{lcccc}
\hline Measures & Baseline T1 & T2 & T3 & T4 \\
\hline Demographic Data Form (DDF) & $\mathrm{X}$ & & & \\
Schwartz Cancer Fatigue Scale (SCFS) & $\mathrm{X}$ & $\mathrm{X}$ & $\mathrm{X}$ & $\mathrm{X}$ \\
Fatigue Impact Scale (FIS) & $\mathrm{X}$ & & & $\mathrm{X}$ \\
Center for Epidemiologic Studies & & & & \\
Depression Scale (CES-D) & $\mathrm{X}$ & & & \\
\hline
\end{tabular}

An advantage of all of the measures was that they were simple, available, selfreport, low cost, and could be completed in a brief administration time. The disadvantage of repeating the SCFS and the FIS measures was the subject may have tired of scoring the repeated measure, not scored accurately, or may have felt obliged to mark the tools in a way to please the investigator. A prepared script to greet and discharge the subjects was used by all persons providing either intervention. After completion of the final assessment all subjects were advised that their involvement in the study was finished. Data Analysis Plan

Quantitative data was coded and entered into a personal computer using SPSS 14.0 by the PI. Demographic data was tabulated and analyzed by descriptive statistics. Frequency distributions and measures of central tendency were calculated for all variables. Frequencies of all the variables were generated to look for outliers. The CES-D baseline data and SCFS had no missing data. Four incorrectly entered numbers in the FIS were corrected and re-entered. An alpha level of .05 was used for all statistical tests. 
Data analysis by aim.

- Aim \#1 (Determine the acceptance rate). The number of possible subjects was reported and compared to the actual number who participated. The reasons for refusal were summarized. (Table 7)

- Aim \#2 (Determine the attrition rate). The number of subjects who complete all measures and the reason why subjects dropped was tabulated and reported.

- Aim \#3 (Describe fatigue over 4 weeks in patients with HCV receiving interferon and ribavirin). Two scales the FIS and SCFS were used to measure fatigue. The FIS was initially analyzed by independent $t$-test using entire group data from baseline and time 4 . Three subscales of FIS, the cognitive, physical, and social dimension were examined for the group by paired $t$-tests for both time points and differences. Fatigue was then measured using the SCFS by analysis of variance (ANOVA) for the overall group.

- Aim \#4 (Compare the level of fatigue in the control and experimental groups). The FIS scale measures at baseline and week 4 in the two groups and were analyzed by $t$-tests for the HT and MT group. Following this, the data were analyzed by using analysis of variance (ANOVA) with the SCFS to test for differences between the two groups, changes over time, and time-by-group interaction for self-reported fatigue.

\section{Protection of Human Subjects}

An overview of subject selection and characteristics. The composition of the sample being studied was volunteer adults diagnosed with chronic $\mathrm{HCV}$ who were receiving a combination of interferon and ribavirin treatment as an outpatient in one 
specific clinic. Subjects needed to have reached 18 years old. Gender and ethnicity data were collected from all subjects who were enrolled in this study. None of these subjects were selected because of any present mental illness, pregnancy, prisoner status, institutionalization, or for any specific characteristic other than their role as described in the study sample.

Inclusion of women, minorities, and children. Recruitment of person of both genders and all races was planned; however, the majority of subjects enrolled were males $(n=14 ; 82 \%)$ and the entire group was $100 \%$ Caucasian. The overall incidence of chronic Hepatitis $\mathrm{C}$ in the general population is higher in males than females. Homogeneity of race was anticipated, as only $3.8 \%$ of the entire population of the province where this study was conducted were visible minorities (Statistics Canada, 2001).

Sources of research material. Measures used were the Demographic Data Form (DDF) asking for basic descriptive information and two self-report questionnaires measuring fatigue, the Fatigue Impact Scale (FIS) and the Schwartz Cancer Fatigue Scale (SCFS). In addition, the Centers for Epidemiologic Studies Depression Scale (CES-D) a self-report questionnaire screening for depressive symptoms was used at baseline. A chart review was conducted to gather data referring to genotype, date at $\mathrm{HCV}$ diagnosis, genotype, current treatment, concurrent medications, and the subject were prescribed during the time of the study, HIV status and current hemoglobin if available. All materials collected were limited to specific research purposes that were outlined in the proposal. 
Potential risks. The greatest potential for harm to subjects was disclosure of identity and private information. Potential psychological increased stress could have developed from completing the instruments. Subject burden of the initial interview, scoring of the paperwork and extra clinic appointments could have been tiring. By agreeing to take part in this study, the subjects in the experimental group and the control group were forfeiting having other energy-based interventions during the period of the study.

Risk management procedures. As required by the facility where the study was conducted, one of the two NPs made the initial patient contact and initially explained the study. If the patient agreed to enroll at that time then one of the two NPs completed the consent. For patient who requested more information or time to decide whether to enroll in the study follow up was made by the PI or RA in a face-to-face meeting. Only the PI, RA or one of the two NPs completed the initial measures, the DDF, SCFS, FIS and CESD during a private meeting with the subject. Confidentiality of the subject's name, identifying characteristics and responses were assured. Data was coded with no names assigned to the study data and kept in a locked cabinet. All individuals providing either intervention were instructed about confidentiality. The patient's physician and the NPs were blinded to which intervention the subject received. All subjects were informed that they were free to withdraw from the study at any time without prejudice to future care.

Potential benefits/compensation. Subjects were informed that might find comfort in participating in the study because they would experience additional attention. The subject was also told that they might find the HT or MT intervention relaxing or may possibly experience a reduction in fatigue. Subjects were not reimbursed for participating 
in this study or for participation time. This study did not involve a commercial sponsor. No medications or blood draws were included in this study. 


\section{Chapter 4}

\section{Results}

The purpose of conducting this study was to investigate the feasibility of a protocol using a Healing Touch intervention to decrease fatigue in patients with $\mathrm{HCV}$ who were being treated with a combination treatment of interferon and ribavirin. In this chapter, the demographic data is described first, followed by a comparison of the HT and MT groups to determine differences in these findings at baseline. Analysis of the CES-D was completed next to determine subjects' levels of depression at baseline, followed by comparison of the HT and MT for differences between the two groups. The CES-D, FIS, and SCFS instruments were tested for reliability. Further results are presented according to each of the four research aims and a comparison of fatigue between program completers and non-completers is included.

Demographic Characteristics

Sample demographic statistics were collected using the Demographic Data Form (DDF) that was designed for this study (see Appendix A). The sample consisted of 17 subjects (14 males and 3 females) who had a mean age of 52 years $(S D=5.8)$ ranging from 43 to 65 years old. On average, subjects had been diagnosed with HCV infection for 7 years (range 1 - 18 years). Duration of interferon therapy averaged 4 months (range 1 12 months). Two subjects reported having no co-morbidities and five subjects were diagnosed with depression. Two subjects were not taking any medications except the 
interferon and ribavirin medication prescribed to treat their chronic HCV. Two subjects did not have current hemoglobin tests available prior to the start of the study. Hemoglobin levels on the remaining 15 subjects ranged from 10.4 to $18 \mathrm{dl} / \mathrm{g}$; therefore, none of the subjects for whom results were available was diagnosed with anemia (i.e., < $10 \mathrm{dl} / \mathrm{g}$ ) at baseline. A list of all co-morbidities, medications used, and available hemoglobin levels was obtained (Appendix E). Of the 17 participants who consented to be in the study, 15 participants completed at least one treatment, and 12 participants completed all treatments. Therefore, 15 participants received some form of treatment (i.e., as treated), but the intention was to treat 17 participants (i.e., intent-to-treat). Table 6 includes a complete description of the demographic findings for all participants as well as those who completed all of the treatments. 
Table 6

Comparison of Demographic Variables of HT and MT groups $(N=17)$

\begin{tabular}{|c|c|c|c|}
\hline \multirow[b]{3}{*}{ Variable } & \multicolumn{2}{|c|}{ Overall Group } & HT \\
\hline & \multicolumn{2}{|c|}{ Consented (Completed) } & Consented (Completed) \\
\hline & Frequency $(n)$ & Percent $(\%)$ & Frequency $(n)$ \\
\hline \multicolumn{4}{|l|}{ Gender } \\
\hline Female & $3(3)$ & $18(25)$ & $3(3)$ \\
\hline Male & $14(9)$ & $82(75)$ & $7(5)$ \\
\hline \multicolumn{4}{|l|}{ Educational Level } \\
\hline Less than secondary & $4(4)$ & $24(33)$ & $3(3)$ \\
\hline Secondary & $6(2)$ & $35(17)$ & $3(2)$ \\
\hline Some post-secondary & $4(3)$ & $24(25)$ & $3(2)$ \\
\hline University Graduate & $3(3)$ & $18(25)$ & $1(1)$ \\
\hline \multicolumn{4}{|l|}{ Race } \\
\hline Caucasian & $17(12)$ & $100(100)$ & $10(8)$ \\
\hline \multicolumn{4}{|l|}{ Marital Status } \\
\hline Single/never married & $2(1)$ & $12(8)$ & $1(1)$ \\
\hline Married/common-law & $7(5)$ & $41(42)$ & $5(4)$ \\
\hline Divorced/separated & $8(6)$ & $47(50)$ & $4(3)$ \\
\hline Widowed & $0(0)$ & $0(0)$ & $0(0)$ \\
\hline \multicolumn{4}{|l|}{ Occupation } \\
\hline Employed & $16(11)$ & $94(92)$ & $9(7)$ \\
\hline Unemployed & $1(1)$ & $6(8)$ & $1(1)$ \\
\hline \multicolumn{4}{|l|}{ Annual Household Income Ranges } \\
\hline Less than $\$ 30,000$ & $9(5)$ & $41(42)$ & $3(3)$ \\
\hline Between $\$ 30,000-\$ 44,999$ & $1(1)$ & $6(8)$ & $2(1)$ \\
\hline Between $\$ 45,000-\$ 59,999$ & $3(2)$ & $18(17)$ & $2(2)$ \\
\hline$\$ 60,000$ or more & $4(4)$ & $24(33)$ & $3(2)$ \\
\hline \multicolumn{4}{|l|}{ Genotype } \\
\hline 1 & $13(8)$ & $77(67)$ & $6(4)$ \\
\hline 2 & $3(3)$ & $18(8)$ & $3(3)$ \\
\hline 3 & $1(1)$ & $6(25)$ & $1(1)$ \\
\hline
\end{tabular}




\section{Comparison of Groups at Baseline}

The two groups were compared at baseline to determine if there were significant differences on specific variables. Analyses were conducted using the DDF, CES-D, FIS, and SCFS findings; no significant differences in subject sociodemographic characteristics, depression, or fatigue were found between the HT and the MT groups at baseline before any of the interventions. Fatigue was measured using two tools to increase the chance of capturing change, the FIS at baseline and Week 4 and the SCFS at baseline and once-a-week 2-day post intervention scheduled times for the following 3 weeks.

Depression was quantified using the 20 -item version of the CES-D. All subjects were given the CES-D to measure presence and severity of depressive symptoms at baseline prior to randomization. All 17 subjects completed the tool with no missing answers. Items $4,8,12$, and 16 were reversed scored. The maximum high score on the CES-D was 60. Outcome scores for the CES-D for the entire sample were determined. The overall group mean was $26(S D=10.99)$ with a range from 7 to 44 . Higher scores on the CES-D indicate higher levels of distress. The majority of subjects $(82 \% ; n=14)$ scored greater than 16 , which is indicative of a clinically significant level of depression that may require further evaluation and treatment.

CES-D baseline scores for the HT and MT were determined. Comparison between the groups showed that the HT group had a mean of $24.60(S D=10.16)$ and the MT group had a mean score of $29.29(S D=16.04)$. Both study groups' mean scores were above 16, which is the level that may suggest clinically significant depression. Next, an independent $t$-test was done to determine whether there was a significant difference in 
depressive symptoms between the HT and MT groups. The independent $t$-test yielded no significant differences in depressive symptoms between the HT and MT groups as measured by the CES-D at baseline $(t=741, d f=15, p=.126)$.

\section{Instrument Reliability}

The reliability of an instrument refers to the extent to which the individual components of that instrument yield the same results over time. Instrument reliability of the CES-D, FIS, and SCFS was evaluated by use of Cronbach's coefficient alpha. Results yielded CES-D $=.88 ; \mathrm{FIS}=.97 ;$ and $\mathrm{SCFS}=.86$ therefore, all of the instruments possessed a high measure of reliability.

Results by Aim

Aim \# 1. Determine the acceptance rate of all possible participants for study inclusion.

Fifty-five patients with a diagnosis of chronic $\mathrm{HCV}$ being treated with a combination of interferon and ribavirin, who met the inclusion criteria for this study, were seen at the hepatology outpatient clinic during the recruitment period from July 2006 to July 2007. All 55 individuals were invited to participate in the study by one of two NPs in a face-to-face meeting at the time of their regular clinic appointment. Seventeen of the 55 individuals approached agreed to participate in the study, yielding an acceptance rate of $31 \%$. The most frequently reported reasons for not participating were travel and distance issues $(61 \% ; n=23)$, followed by stress/family issues $(18 \% ; n=7)$. A complete list of the reasons for refusal is summarized in Table 7. 
Table 7

Subject Refusal

\begin{tabular}{lcc}
\hline \multicolumn{1}{c}{ Reason } & Frequency & Percent \\
\hline Travel/Too far a distance & 23 & 61 \\
Stress/family issues & 7 & 18 \\
Work commitment & 4 & 11 \\
Finances & 2 & 5 \\
Involved in too much prior research & 2 & 5 \\
\hline
\end{tabular}

Aim \# 2. Determine the attrition rate of all participants enrolled in the study.

The attrition rate was determined followed by a description of the subjects' reasons for withdrawal. The attrition rate was $29 \%$. Of the 17 individuals who initially agreed to participate, $71 \%(n=12)$ completed all measures and $29 \%(n=5)$ withdrew at different stages of the study. Two subjects dropped out from the HT group and three subjects were from the MT group, but this difference was not significant $\left(\chi^{2}=1.04, p=\right.$ .31). All 17 subjects completed the baseline measures and were randomized to treatment prior to any withdrawal.

Of the $11 \%(n=2)$ of subjects in the HT group who did not complete the study, one withdrew following enrollment before scheduling of the first intervention and reported being too sick to continue. The other subject from the HT group withdrew prior to the third intervention and reported being too busy at work to continue in the study.

In the MT group, $18 \%(n=3)$ of subjects did not complete the study. One subject completed all the baseline measures and withdrew before receiving any intervention. 
During the phone call to schedule this subject's first intervention, he reported being too sick to continue. One subject, who did not return to the clinic for the third intervention, declined a new appointment and declined to give a reason for dropping out of the study. Another subject was a no-show for the final intervention. Two attempts by phone to reschedule an appointment were unsuccessful, as the subject again did not keep the new appointment. This subject reported deciding to withdraw from the study because of travel, parking expenses, and the inconvenience of trips to the clinic site. There was no pre-established protocol to allow for a missed session and to remain in the study.

Of the five subjects who withdrew from the study, four made less than $\$ 30,000$ a year, four subjects had been on the interferon and ribavirin treatment for a month or less, and two subjects had three co-morbidities. All five subjects who did not complete the study were male.

Data obtained from all subjects were included in study analyses because of the small number of subjects enrolled in the study. Only baseline findings relate to all 17 subjects; however, a comparison of the demographic information for those who consented and those who completed treatment can be found in Table 6.

Aim \# 3. Describe fatigue over 4 weeks in patients with HCV receiving interferon and ribavirin.

Two scales, the Fatigue Impact Scale (FIS) and the Schwartz Cancer Fatigue Scale (SCFS) were used to measure fatigue over time. The FIS scale was analyzed first.

The FIS was a self-report scale that asked subjects to recollect their fatigue impact for the previous month. The range of scores possible on the FIS scale was from 0 to 160 , with lower scores representing less impact of fatigue. Fatigue impact was quantified 
using scores obtained from the FIS at two points in time, baseline prior to randomization and at Week 4 after completion of all interventions. All 17 subjects completed the FIS at baseline; however, due to attrition, only 12 subjects completed both measures.

The overall sample mean score of fatigue decreased over time as measured by the FIS from $86.94(S D=32.42, n=17)$ to $73.67(S D=36.67, n=12)$. This represented an average improvement of 13.27 points and indicated that there was a trend of lower impact of fatigue on subjects over time. However, the average baseline scores for those participants who completed the program was 78.92 , only 5.25 points higher than at program completion. A paired $t$-test was run to determine the overall group difference between the FIS at baseline and at Week 4. The $t$-test revealed that there were no significant differences between the fatigue levels for the two time points.

The FIS was further analyzed by computing the overall group scores on three separate domains: cognitive (10 items), physical (10 items) and social (20 items). Maximum possible scores were 40,40 , and 80 respectively. As seen in Table 8, the results revealed that the overall group mean of the impact of fatigue over time was modestly affected in the cognitive dimension but decreased (i.e., was improved) in the physical and social dimensions. 
Table 8

Pre-Post Fatigue Impact Scale (FIS) Scores $(n=12)$

\begin{tabular}{lccccc}
\hline Domain & Baseline & Week 4 & Difference & $t$ & $p$ \\
\hline Cognitive & 16.08 & 15.92 & -.17 & .053 & .959 \\
Physical & 25.50 & 22.42 & -3.08 & 1.009 & .335 \\
Social & 37.33 & 35.33 & -2.00 & .324 & .753 \\
Total & 78.92 & 73.67 & -5.25 & .439 & .669 \\
\hline
\end{tabular}

Since the baseline scores were noticeably different, a comparison was done of the completers and non-completers. Every subscale score and the total score was worse for non-completers at baseline; however, this difference failed to achieve statistical significance (Table 9). 
Table 9

Differences between Program Completers and Non-Completers in the FIS

\begin{tabular}{lccccc}
\hline Domain & Non-Completer & Completer & Difference & $t$ & $p$ \\
\hline Cognitive & 22.80 & 16.08 & 6.72 & 1.380 & .188 \\
Physical & 30.60 & 25.50 & 5.10 & 1.353 & .196 \\
Social & 52.80 & 37.33 & 15.47 & 1.715 & .107 \\
Total & 106.20 & 78.92 & 27.28 & 1.667 & .116 \\
\hline
\end{tabular}

The Schwartz Cancer Fatigue Scale (SCFS) was the second measure used to evaluate fatigue. This self-report questionnaire measured how fatigue made the subject feel in the previous 2 or 3 days. Possible scores ranged from 6-30 with higher scores indicating higher presence of fatigue symptoms. Data were collected at baseline and weekly 2 to 3 days after each intervention for a consecutive 4-week period.

The SCFS data were collected from 17 subjects at Time 1,15 subjects at both Time 2 and Time 3, and 12 subjects at Time 4 . At the end of the study, there were 12 complete sets of SCFS data. Individual scores were determined by summing the data and frequencies were run for the overall group. Descriptive analyses indicated that the overall groups' mean fatigue decreased steadily over time from 18.18 at baseline to 14.83 at Week 4 (see Table 10). 
Table 10

SCFS Descriptive Statistics for Overall Group and Time

\begin{tabular}{lcccc}
\hline Interval & Mean & $S D$ & $F$ & $p$ \\
\hline Week 1 & 18.18 & 4.14 & 1.128 & .342 \\
Week 2 & 17.13 & 4.85 & & \\
Week 3 & 16.07 & 5.68 & & \\
Week 4 & 14.83 & 5.70 & & \\
\hline
\end{tabular}

Program completers scored lower $(M=17.58)$ in the SCFS than non-completers $(M=19.60)$ at baseline. While program non-completers demonstrated a higher level of fatigue, this difference was not statistically different $(t=.910, p=.377)$.

Aim \# 4. Compare the level of fatigue in the control and experimental groups.

Both the FIS and SCFS measures were used to test for differences in fatigue between the HT and MT group over time. The FIS baseline data for the treatment and control group were analyzed first to determine whether there was a difference in fatigue between the HT and MT group at Week 1 prior to randomization of subjects to groups. Descriptive analysis were run and results indicated the Week 1 grand means for fatigue were $82.50(S D=31.47)$ for the HT group and slightly higher at $93.29(S D=35.17)$ for the MT group. An independent samples $t$-test for the equality of means was performed $(t$ $=0.66, d f=15, p=.52$ ), indicating no significant difference between the HT and MT group on fatigue at Week 1. The Week 4 mean was $69.00(S D=41.78)$ for the HT group and $83.00(S D=26.17)$ for the MT group. Analysis by a separate independent samples $t$ test at Week 4 revealed no significant difference in fatigue between the HT or MT groups 
$(t=0.61, d f=10, p=.56)$. The means of both the HT and MT group decreased from Week 1 to Week 4, indicating the subjects in both groups had a decline of fatigue over time; however, a statistically significant difference in fatigue did not differentiate the two groups (Table 11).

Table 11

FIS Comparison of Domains between Groups by Time

\begin{tabular}{lcccc}
\hline & \multicolumn{2}{l}{ Healing Touch (HT) } & \multicolumn{2}{c}{ Mock Touch (MT) } \\
\hline & Week 1 & Week 4 & Week 1 & Week 4 \\
Grand Means & 82.50 & 69.00 & 93.29 & 83.00 \\
Cognitive & 18.10 & 16.38 & 18.00 & 15.00 \\
Physical & 25.20 & 19.50 & 29.57 & 28.25 \\
Social & 39.20 & 33.13 & 45.71 & 39.75 \\
\hline
\end{tabular}

Data from the SCFS, which measured fatigue symptoms in each subject from the HT and MT groups at four different times were analyzed to look for change-by-group over time. As shown in Table 12, the HT mean group had a modest increase at Week 2 and then a steady decline in the level of fatigue over the following 2 weeks. Mean fatigue in the MT group decreased over the first 3 weeks followed by a modest rise at Week 4. 
Table 12

SCFS Descriptive Statistics by Group and Time

\begin{tabular}{lllll}
\hline & \multicolumn{2}{c}{ Healing Touch } & \multicolumn{2}{c}{ Mock Touch } \\
\hline Interval & $\underline{\text { Mean }}$ & $\underline{\text { S.D }}$ & $\underline{\text { Mean }}$ & $\underline{\text { S D }}$ \\
Week 1 & 16.87 & 4.05 & 19.00 & 5.16 \\
Week 2 & 17.00 & 4.34 & 18.50 & 5.26 \\
Week 3 & 15.50 & 6.71 & 15.25 & 4.99 \\
Week 4 & 14.38 & 6.14 & 15.75 & 5.44 \\
\hline
\end{tabular}

A general linear model two-way analysis of variance (ANOVA) was done to test whether group and/or session had any effect on subjects' rating of the symptoms of fatigue. ANOVA was selected over $t$-tests because repeated $t$-tests inflate the Type 1 error (Huck, 2007). In an ANOVA, the dependent variable is quantitative and the factors are categorical. The assumptions for ANOVA include random sample and equal cell variances. While the data should be symmetrical, ANOVA is robust as it relates to departures from normality (SPSS, Inc, 2006). Levene's test of equality was not significant $(F[7,51]=0.49, p=.84)$ suggesting that equality of variances of the two groups for fatigue scores over the four time points could be assumed. Two-way interaction of group and session neither revealed significant differences in fatigue ( $F[3$, $51]=0.12, p=.95)$, nor were the main effects for $\operatorname{session}(p=.42)$ or group $(p=.22)$ statistically significant. 
Table 13

SCFS ANOVA Results

\begin{tabular}{|c|c|c|c|c|c|}
\hline Source & $\begin{array}{c}\text { Type III Sum of } \\
\text { Squares }\end{array}$ & $d f$ & $\begin{array}{l}\text { Mean } \\
\text { Square }\end{array}$ & $F$ & Sig. \\
\hline Corrected Model & 137.611(a) & 7 & 19.659 & .728 & .649 \\
\hline Intercept & 14898.373 & 1 & 14898.373 & 551.371 & .000 \\
\hline Session & 78.524 & 3 & 26.175 & .969 & .415 \\
\hline HT & 42.567 & 1 & 42.567 & 1.575 & .215 \\
\hline Session * HT & 9.720 & 3 & 3.240 & .120 & .948 \\
\hline Error & 1378.050 & 51 & 27.021 & & \\
\hline Total & 18027.000 & 59 & & & \\
\hline Corrected Total & 1515.661 & 58 & & & \\
\hline
\end{tabular}

Summary

In this chapter, each of the four aims was examined and the results presented. There were no baseline differences between the HT and MT group. None of the results were statistically significant. Healing Touch did not significantly decrease the impact of fatigue or fatigue scores measured by the FIS and SCFS in patients diagnosed with HCV who were being treated with interferon and ribavirin. Discussion and implications related to the findings will follow in Chapter 5. 
Chapter 5

\section{Discussion and Conclusion}

The primary purpose of this study was to test the feasibility of a protocol using a HT intervention for fatigue in patients with $\mathrm{HCV}$ who were being treated with a combination therapy of interferon and ribavirin. This study was informative as it was the first to investigate the use of HT in adults with HCV. Specific areas of interest were sample acceptance, refusal rates, attrition rates, measurements of subject fatigue over a 4week period, and comparison of fatigue between those subjects receiving HT and those receiving MT treatment. The discussion of the findings is organized according to issues related to methodology and the results followed by implications for nursing practice, nursing education and nursing research.

\section{Issues Regarding Methodology and Results}

In this study, the treatment-related effect of HT on fatigue was not found to be statistically significant. The findings did not add statistical support for Rogers' science of unitary human beings, the theoretical framework guiding this study; however, that could be explained by the small sample size. Participants receiving HT reported actual decreases in the impact of fatigue, suggesting clinical significance even in the absence of statistical findings.

Recruitment for this study was more challenging than anticipated. Every possible individual who met the study criteria from this hepatology clinic was approached and 
invited to participate by a face-to-face meeting with one of the two NPs employed in the clinic. Of the 55 patients who met the criteria and attended the clinic during the 12 months that the study conducted, $31 \%(n=17)$ agreed to participate. A higher response rate was expected based on previous studies completed with the HCV population at this clinic (Hirsh, et al., 2001; Vallis, Hirsch, Patel, Johnstone, \& Peltekian, 2001, White et al, 2007). Although the location of this study was a busy hepatology clinic, both nurse practitioners were able to contact and maintain a list of all persons meeting the entrance criteria, each individual contacted, and a list of reasons for refusal. Seventy-nine percent of the reason given for refusal to join the study was accounted for by travel $(61 \%)$ and family stress $(18 \%)$. Other factors reported were work commitment, finances, and too much previous research. Approximately $60 \%$ of the population of this province lived in rural areas and, for many individuals, the distance to the clinic was well over 200 miles. The decision was made to use one site for all interventions in order to reduce the possibility of differing environments that might have influenced the results. One explanation for the difficulty in recruiting subjects might have been that this study involved three extra return trips to the clinic to receive either intervention while previously noted studies at this site were completed during the regular clinic visit. Similarly, in a larger study examining the use of HT, therapeutic massage, and presence in patients with cancer, with a consent rate of $42 \%$, and travel and transportation issues ( $26 \%$ ) were reported as the most prevalent reasons for refusal (Post-White et al., 2003).

The overall attrition rate was $29 \%$. Of the 17 individuals who initially agreed to participate in this study, $71 \%(n=12)$ completed all measures and $29 \%(n=5)$ withdrew 
at different stages of the study. All 17 subjects completed all of the baseline measures and were randomized to treatment. The attrition rate was almost equal for both groups, with two subjects from the treatment group and three subjects from the control group withdrawing. A pattern of attrition occurred prior to Week 2 and again prior to Week 4 . Prior to Week 2, one subject from each group completed the baseline measures and then, before scheduling of the first intervention, withdrew and reported being too sick to continue in the study. The time of greatest attrition was prior to the final intervention, with two subjects in the control group and one subject in the treatment group withdrawing, reportedly being too busy with work. One of the control subjects declined to give a reason and the other control subject reported travel, parking expenses, and the inconvenience of visiting the clinic. Parking at the clinic was expensive, limited, and least available during the times of day when intervention appointments were available. Four of the five subjects who withdrew were in the first month of interferon and ribavirin treatment. One possible explanation for their withdrawal is that reported side effects of interferon and ribavirin combination therapy were inclined to be more severe in the initial weeks of treatment (Strader et al, 2004). Neither of the fatigue measures had differing attrition patterns; however, those subjects who withdrew had higher levels of fatigue compared to those who completed the study. Four of the five subjects who withdrew had baseline FIS fatigue scores in the top $30 \%$ of the overall group. Similarly, Post-White et al. (2003) reported a $29 \%$ attrition rate in a larger study of HT.

In this study with a small sample size, the HT group reported greater declines in fatigue in the physical and social domains than the MT group as measured by the FIS. None of the results were statistically significant. Possible explanations for this may be 
that the HT intervention did not work, was not adequate in terms of frequency or duration, was not measured close enough to the post-intervention to effect a change, or, most likely, the small sample size would have require an extraordinary change in order to detect statistical significance. Using both the FIS and SCFS, the findings support the presence of fatigue in individuals treated with combination interferon and ribavirin for chronic HCV.

There were no previous studies investigating the use of HT or any energy therapy for fatigue in the HCV population. The three studies that measured fatigue and a HT intervention reported conflicting results. Rexilius (2002) measured an increase in fatigue in caregivers of patients undergoing autologous hematopoietic stem cell transplant using the Multidimensional Fatigue Inventory-20. Post-White et al. (2003) reported a slight decrease in fatigue in patients with cancer who were receiving chemotherapy using the POMS. Wardell (2005) reported a decline in fatigue using the POMS in patients with chronic neuropathic pain. Perhaps the POMS better captures the dimensions of fatigue affected by HT than the FIS and SCFS measures used in this study.

Major problems in measuring and treating fatigue were that the underlying mechanism remains unknown and fatigue is a self reported symptom (Jones, 2004). Self reported data may be susceptible to bias. Based on the data collected, individuals with chronic $\mathrm{HCV}$ were able to complete both of the fatigue instruments without difficulty. The FIS relied on a 1-month recollection of the impact of fatigue; individuals might not have been able to recall past behaviors or feelings accurately over the past month. The SCFS was a recollection of fatigue for the past 2-3 days; individuals might have filled out the questionnaire on a day other than the designated one. The completed SCFS form was 
returned by the individual at the next weekly visit or completed over the phone with the PI or RA. It could be that there were immediate effects not captured by the instrument because it did not request measurement until 2-3 days post intervention. Perhaps measures of the immediate effects of the HT need to be measured closer to the intervention as suggested by Dowd et al. (2007).

Using this protocol the MD and NPs remained blinded to the group assignment of the subject for the duration of the study. No negative effects of the HT intervention were reported and one patient requested a referral to a HT practitioner after the completion of the study.

In order to measure fatigue in the HT against the MT group, it was necessary to find individuals willing to provide the HT and MT intervention. The benefit of using a number of different practitioners to provide the $\mathrm{HT}$ and MT interventions was the possible influence of only one provider was decreased. Both the HT and MT interventions were standardized through a face-to-face class, demonstration, practice session, and written instructions. The PI did not provide any of the interventions, which was previously reported as a possible conflict.

The HT practitioners were recruited through the local HT community group. Several meetings were held to explain the study to all members of the group and to recruit volunteer practitioners who met the study criteria. All HT practitioners involved in the study were present or previous employees of the medical center where the study was conducted.

In the stage of study planning, the significant challenge of recruiting appropriate individuals as MT providers was not recognized in advance. The MT providers had to 
meet certain criteria and be approved by the facility to gain access and interact with the patients in the hospital environment. In addition, they needed a strong understanding of the importance of patient confidentiality. Another requirement was having free time available to volunteer during normal work hours as well as attending a class to learn and practice the MT intervention. Several individuals approached as MT providers reported they believed providing a placebo intervention was unethical. The three volunteer individuals who were finally recruited to provide the MT intervention were all practicing or retired nurses from the facility. The challenge of recruiting sham providers in this study was consistent with results described by Slater (1995). In that study, several of the sham HT providers gave one treatment and then dropped out of the study, each study site had to train several sham providers just to provide a few interventions and one site had equal numbers of sham providers and study subjects. Slater noted that the sham providers reported feeling silly or awkward while providing the intervention. In both Slater's work and this present study the sham providers were RNs.

In this study, none of the practitioners from either group knew any of the subjects prior to the study. Subjects were scheduled individually for their intervention sessions to decrease the possibility of contamination between groups in the waiting room. If there had been a previous interaction between the practitioner and the subject, or between subjects it might have influenced the results. A future study protocol should be revised to prevent interaction in practitioner/subject dyads who have previously known each other.

Small sample size is the major limitation of this study. Participant enrollment continued for 12 months but a less than anticipated sample size was obtained. Potential participants who did not enroll in the study identified barriers of travel, family stress, 
work commitment, finances and too much previous research. Travel and family stress were the primary concerns and were outside of the control of this study. All intervention treatment visits were only available during normal work hours, which could explain work commitment concerns as a reason for refusal. Subjects enrolled in the study were able to schedule appointments at a suitable time despite the fact that they were employed and the clinic was only available during regular work hours. Perhaps those individuals who refused to enter the study had lower income levels than those who participated so that expenses related to travel were a greater concern. Issues of too much previous research by potential participants add to the challenge of recruiting and retaining individuals in this specific population.

The demographics of the sample obtained generally approximated the population of patients with $\mathrm{HCV}$ on interferon and ribavirin treatment who attend this clinic in terms of race (100\% Caucasian) and gender ( $82 \%$ male). Inferences will not extend to the larger group of individuals approached but only to individuals similar to those who supplied the data.

Implications for nursing practice

The purpose of this pilot study was to test the feasibility of a protocol for HT fatigue in patients with chronic HCV who were being treated with interferon and ribavirin. The individuals enrolled in this study had increased levels of fatigue and depression consistent with that reported in previous studies. Although no results were statistically significant, the impact of fatigue was lessened in the HT group compared to the MT group. This finding may influence the use of HT in future nursing practice. 
Implications for Nursing Education

HT is being taught in nursing education and is approved and endorsed by professional nursing associations, a fact that challenges nurse educators to provide a fuller understanding of HT to support this as a credible area of study. Healing Touch is presently being taught as a nursing intervention recommended for use with the nursing diagnosis, disturbed energy field.

Implications for Nursing Research

Preliminary information gained from this pilot study may be applicable when considering a protocol to study the effects of $\mathrm{HT}$ on fatigue in patients with chronic $\mathrm{HCV}$ who are receiving interferon and ribavirin therapy. This study served to highlight factors why individuals with chronic $\mathrm{HCV}$ being treated with interferon and ribavirin do not enroll or once enrolled withdraw from such a study. Use of an experimental randomized controlled design and expanding the study for a longer duration with a larger sample size is proposed for future studies. Since travel and family stress were the highest reasons given by individuals for not participating, suggestions for future research include participant reimbursement for travel expenses, having interventions provided by home visits, or using multiple sites for recruitment.

Recommendations to maintain sample size by limiting subject attrition include changing the protocol to include weekly supportive phone calls by the PI or RA to each subject. In this study, the findings revealed that 4 of the 5 subjects who withdrew were in the first month of interferon and ribavirin treatment. The first month of this combination treatment is considered to have the most severe side effects so postponing enrollment until one month after initiation of treatment may result in decreased attrition. In addition, 
revising the protocol to allow subjects to miss visits and remain in the study may increase sample size of a future study.

It was possible that the timing of the HT interventions may not have been frequent enough to influence changes in levels of fatigue. In future studies choosing a different HT intervention schedule may reveal different results. The underlying mechanism of HT is not understood and further studies are needed to make recommendations for dosage and length of effects.

Based on findings of this study, a future randomized controlled trial using the instruments piloted in this study is feasible. All instruments were easily completed by subjects in the allotted time. The instruments were easy to administer and score. In future studies using the FIS scale as the primary evaluation tool to capture changes in fatigue should be considered.

A strong correlation of fatigue and depression has been previously reported (Bacon, 2004; Zickmund et al., 2006). In this study, depression was assessed at baseline but not over time. Baseline findings revealed $82 \%(n=14)$ of subjects had levels of depression above 16 as measured by the CES-D. This level of depression is considered worthy of further clinical investigation. In a future study, because of the strong association of depression and fatigue, it is recommended that depression be assessed at baseline and at the end time point.

In this study, the influence of medications use and outside life circumstances that may have contributed to the subjects fatigue was not assessed. Several of the subjects' prescribed medications such as antidepressants, noted in the current medication list have fatigue as a possible side effect (Appendix E). The addition of a daily self-report diary 
that would include prescribed medication, over the counter drugs, reductions in routine daily medications and herbals use is recommended.

Another suggestion to strengthen a future study protocol is inclusion of an exit interview with the subject just prior to completion of the study. Questions to include in this interview would relate to life style changes during the study, changes in health status particularly related to their HCV status, perceived benefits or dislikes of the intervention, and a subjective description of fatigue. Information obtained from this interview would provide data that could be qualitatively analyzed to provide insight into the participants' perceptions. Addition of a qualitative component may provide data that has significance for individuals with chronic $\mathrm{HCV}$ who experiencing fatigue while receiving interferon and ribavirin.

\section{Conclusion}

In conclusion, it is feasible to recruit patients with chronic $\mathrm{HCV}$ who are being treated with interferon and ribavirin, for a study with this protocol to investigate the use of HT to treat fatigue. Small sample size is the major limitation of this study. Present available treatments for fatigue in this population are of mixed and limited benefit, so investigations of new and novel therapies are required. These preliminary data indicate the need for a larger future study to evaluate the effectiveness of HT and weighs its benefit in treating fatigue in patients with chronic $\mathrm{HCV}$ receiving interferon and ribavirin. Results from such investigations might change the use of $\mathrm{HT}$, which is non-invasive, low tech, low cost intervention that may be integrated into practice with traditional treatment. 


\section{References}

Arase, Y. (2006). Side effects of interferon therapy for chronic hepatitis C. Nippon Rinsho. 64(7), 1363 - 1367.

Bacon, B. R. (2004). Managing hepatitis C. American Journal of Managed Care, $10(2$ Suppl, S30-40.

Bailey, A. (1998). Esoteric Healing. New York: Lucis Press.

Barkhuizen, A., Rosen, H. R., Wolf, S., Flora, K., Benner, K., \& Bennett, R. M. (1999). Musculoskeletal pain and fatigue are associated with chronic hepatitis C: a report of 239 hepatology clinic patients. American Journal of Gastroenterology, 94(5), $1355-1360$.

Barnes, P.M., Powell-Griner, E., McFann, K., \& Nahin, R.L. (2004). Complementary and alternative medicine use among adults: United States, 2002. Advance Data, 343, $1-19$.

Benson, L., Birkel, A., Caldwell, L., Stafford-Fox, V., \& Casarico, B. (2000). Advances in the treatment of hepatitis $\mathrm{C}$ : combination antiviral therapy with interferon alfa$2 \mathrm{~b}$ and ribavirin. Journal of American Academy of Nurse Practitioners, 12(9), 364-373.

Birkel, A., Caldwell, L., Stafford-Fox, V., Casarico, B., \& Benson, L. (2000). Combination Interferon alfa-2b/ribavirin therapy for the treatment of hepatitis $\mathrm{C}$ : nursing implications. Gastroenterology Nursing, 23(2), 55-62.

Brennan, B.(1988) Hands of Light. New York: Bantam.

Bruyere, R. (1994). Wheels of Light. New York: Fireside. 
Center for Disease Control. (2008, March). Viral Hepatitis $C$ fact sheet. Retrieved February 19, 2009 from Website: http://www.cdc.gov/ncidod/diseases/hepatitis/c/fact.htm

Clark, C. H., Mahoney, J. S., Clark, D. J., \& Eriksen, L. R. (2002). Screening for depression in a hepatitis $\mathrm{C}$ population: the reliability and validity of the Center for Epidemiologic Studies Depression Scale (CES-D). Journal of Advanced Nursing, $40(3), 361-369$.

Crone, C. C., Gabriel, G. M., \& Wise, T. N. (2004). Managing the neuropsychiatric side effects of interferon-based therapy for hepatitis C. Cleveland Clinic Journal of Medicine, 71 Supplement 3, S27-32.

Davis, G. L., Beck, J. R., Farrell, G., \& Poynard, T. (1998). Prolonged treatment with interferon in patients with histologically mild chronic hepatitis C: a decision analysis. Journal of Viral Hepatitis, 5(5), 313-321.

Dowd, T., Kolbaca, K., Steiner, R. \& Fashinpaur, D. (2007). Comparison of a Healing Touch, coaching and a combined intervention on comfort and stress in younger college students. Holistic Nursing Practice. 21(4), 194-202.

Eisenberg, D.M., Davis, R.B., Ettner, S.L., Appel, S., Wilkey, S., van Rompay, M., et al. (1998). Trends in alternative medicine use in the United States, 1990-1997: Results of a follow-up national survey. Journal of the American Medical Association, 280, 1569-1675.

Fisk, J. D., Ritvo, P. G., Ross, L., Haase, D. A., Marrie, T. J., \& Schlech, W. F. (1994). Measuring the functional impact of fatigue: initial validation of the fatigue impact scale. Clinical Infectious Disease, 18 Supplement 1, S79-83. 
Frederick-Rust, M., Zeuzem, S., \& Sarrazin, C. (2007). Current Therapy for Hepatitis C. International Journal for Colorectal Disease, 22(4) 341-149.

Fried, M. W. (2002). Side effects of therapy of hepatitis C and their management. Hepatology, 36(5 Supplement 1), S237-244.

Fried, M. W., Shiffman, K.R., Reddy, C., Smith, G., Marionos, F.L., Goncales, Jr. D. et al. (2002).Peginterferon alfa-2 plus ribavirin for chronic hepatitis $\mathrm{C}$ viral infection. New England Journal of Medicine, 347(13), 975-982.

Geddes, N.J. (1999). The experience of personal transformation in healing touch (HT) practitioners: A hueristic inquiry (unitary paradigm). (Doctoral dissertation, Virginia Commonwealth University, 1999.)_Dissertation Abstracts International, AAT 9935722

Glacken, M., Coates, V., Kernohan, G., \& Hegarty, J. (2003). The experience of fatigue for people living with hepatitis C. Journal of Clinical Nursing, 12(2), 244-252.

Haahr, M. (2006). Random integer generator. Retrieved January 22, 2006, from http://www.random.org/integers/

Hassoun, Z., Willems, B., Deslauriers, J., Nguyen, B. N., \& Huet, P. M. (2002). Assessment of fatigue in patients with chronic hepatitis $\mathrm{C}$ using the Fatigue Impact Scale. Digestive Diseases Sciences, 47(12), 2674-2681.

Hadziyannis, S. J., Sette, H. Jr., Morgan, T. R., Balan, V., Diago, M., Marcellin, P., et al. (2004). Peginterferon-alpha2a and ribavirin combination therapy in chronic hepatitis C: a randomized study of treatment duration and ribavirin dose. Annals of Intern Medicine, 140, 346-355. 
Healing Touch Level 1 Notebook (2007). Healing Touch Program. Golden, CO. ISBN13:978-0-9790477-2-5

Hirsch, G., Adams, F., Peterson, T. C., Johnstone, K., Patel, S., \& Peltekian, K. M. (2001). In Hepatitis C Virus (HCV) Infected Individuals Attending a Multidisciplinary Liver Clinic, Complimentary and Alternative Medicine (CAM) Use is Associated with Higher Level of Education. Paper presented at the Canadian Liver Conference, Montreal Canada.

Hover- Kramer, D., (2001). Healing Touch: A Guide Book for Practitioners. Albany, NY: Delmar.

Huber, D. (2001). Does the "C" in hepatitis C stand for complex? Gastroenterology Nursing, 24(3), 120-126; quiz 127-128.

Huck, S. W. (2007). Reading Statistics and Research. (5 $5^{\text {th }}$ ed.). Boston: Allyn \& Bacon Inc.

Jones, E. A. (2004). Fatigue complicating chronic liver disease. Metabolic Brain Disease, 19(3-4), 421-429.

Joy, B. (1979). Joy's Way. New York: Tarcher.

Kallman, J., O'Neill, M., Larive, B., Bopari, N., Calabrese, L., \& Younossi, Z. (2007). Fatigue and Health Related Quality of Life (HRQL) in Chronic Hepatitis C Virus Infection. Digestive Diseases \&Sciences.52(10), 2532-2539.

Kjaergard, L., Krosgaard, K.; \& Gluud, C., K.; \& Gluud, C. (2002). Ribaviran with or without alpha interferon for chronic hepatitis C. The Cochrane Database if Systematic Reviews, CD002234. 
Krucoff, M. W., Crater, S. W., Green, C. L., Maas, A. C., Seskevich, J. E., Lane, J. D., et al. (2001). Integrative noetic therapies as adjuncts to percutaneous intervention during unstable coronary syndromes: Monitoring and actualization of noetic training (MANTRA) feasibility pilot. American Heart Journal, 14(5), 760-769.

Loveland Cook, C., Guerrerio, J., \& Slater, V. (2004). Healing touch and quality of life in women receiving radiation treatment for cancer: A randomized controlled trial. Alternative Therapies, 10(3), 34-41.

Malinski, V. (1986). Explorations on Martha Rogers' science of unitary human beings. Norwalk, CT: Appleton-Century-Croft.

Nanda-1. (2007) Nursing diagnoses: Definitions and classifications 2007-2008. Philadelphia: NANDA International.

McDonald, J., Jayasuriya, J., Bindley, P., Gonsalvez, C., \& Gluseska, S. (2002). Fatigue and psychological disorders in chronic hepatitis C. Journal of Gastroenterology and Hepatology, 17(2), 171-176.

McHutchison, J. G., Mannis, M., Patel K, et al., for the International Hepatitis Interventional Therapy Group. (2002). Adherence to combination therapy enhances sustained response in genotype- 1 infected patients with chronic hepatitis C. Gastroenterology, 123, 1061-1069.

Piche, T., \& Tran, A. (2001). Fatigue in patients with chronic hepatitis C: a sign to look for. Gastroenterology Clinical Biology, 25(12), 1059-1060.

Piche, T., Vanbiervliet, G., Cherikh, F., Antoun, Z., Huet, P., Gelsi, E. et al. (2005). Effect of ondansetron, a 5- HT3 receptor antagonist on fatigue in Hepatitis C: a randomized double blind, placebo controlled study. Gut. 54(10), 1169-1173. 
Poynard, T., Cacoub, P., Ratziu, V., Myers, R. P., Dezailles, M. H., Mercadier, A., et al. (2002). Fatigue in patients with chronic hepatitis C. Journal of Viral Hepatitis, 9(4), 295-303.

Post-White, J.; Kinney, M.; Savik, K.; Gau, J.; Wilcox, C. \& Lerner, I. (2003). Therapeutic massage and Healing Touch improve symptoms in cancer. Integrative Cancer Therapies. 2(4) 332-344.

Rasenack, J., Zeuzem, S., Feinman, S. V., Heathcote, E. J., Manns, M., Yoshida, E. M., et al. (2003). Peginterferon alpha-2a (40kD) [Pegasys] improves HR-QOL outcomes compared with unmodified interferon alpha-2a [Roferon-A]: in patients with chronic hepatitis C. Pharmacoeconomics, 21(5), 341-349.

Rexilius, S. J.; Mundt, C. A.; Megel, M. E., \& Agrawal, S. (2002). Therapeutic effects of massage therapy and healing touch on caregivers of patients undergoing autologous hematopoietic stem cell transplant. Oncology Nursing Forum, 29, 3.

Richardson, M. A., \& Straus, S. E. (2002). Complementary and alternative medicine: Opportunities and challenges for cancer management and research. Seminars in Oncology, 29, 531-545.

Rogers, M. E. (1970). An introduction to the theoretical basis of nursing. Philadelphia: F.A.Davis.

Rogers, M. E. (1992). Nursing science and the space age. Nursing Science Quarterly, $5(1), 27-34$.

Seeff, L. B., \& Hoofnagle, J. H. (2002). National Institutes of Health Consensus Development Conference: Management of hepatitis C. Hepatology, 36(5, Supplement 1) S1-2. 
Schaefer, K. M. (2004). Persons with hepatitis C experienced fatigue as being multidimensional with severity dependent on intensity, duration, and frequency. Evidenced Based Nursing, 7(1), 32.

Shadish, W. R., Cook, T. D., \& Campbell, D. T. (2002). Experimental and quasiexperimental designs for generalized causal inference. New York: Houghton Mifflin.

Sherman, M., Shafran, S., Burak, K., Doucette, K., Wong, W. et al. (2007). Canadian consensus guidelines management of chronic hepatitis C. Canadian Journal of Gastroenterology, 12(Suppl C June), 25C-34C

Silva, M. A. C. (1995). The effect of relaxation touch on the recovery level of postanesthesia abdominal hysterectomy patients. Unpublished doctoral dissertation. Texas Women's University, Texas

Slater, V. E. (1996). Safety, elements, and effects of healing touch on chronic nonmalignant abdominal pain. (Doctoral dissertation), University of Tennessee, 1996). Dissertation Abstracts International. 57 (07B) 4303.

SPSS, Inc. (2006). SPSS 14.0 for Windows student version (computer software). Chicago.

Strader, D. B., Wright, T., Thomas, D. L., \& Seeff, L. B. (2004). AASLD practice guidelines. Diagnosis, management, and treatment of hepatitis C. Hepatology, 39(4), 1147-1171.

Statistics Canada. (2001 Census). Visible minority population, by province and territory. Retrieved Oct.31, 2008 from Website: http://www.statcan.gc.ca 
Taylor, B. (2001). The effects of healing touch on the coping ability, self esteem and general health of undergraduate nursing students. Complementary Therapies in Nursing and Midwifery, 7(1), 34-42.

Terrault, N. A. (2004). Update on the treatment of chronic viral hepatitis. Gastroenterology Clinics, 33(3).

Vallis, T. M., Hirsch, G., Johnstone, K., \& Peltekian, K. M. (2001, May 1). Mood, fatigue and affective states in hepatitis $C$ virus $(H V C)$ infected individuals attending a multidisciplinary liver clinic. Paper presented at the 1rst Canadian Conference on Hepatitis C, Montreal Quebec.

Vallis, T. M., Peltekian, K., \& Hirsh, G. (2003). Fatigue and quality of life in hepatitis C: Fact or fiction? Journal of Clinical Psychology in Medical Settings, 10(4). 267271.

Van Aken, R. (2004). Emerging from depression: the experiential process of healing touch for people with moderate depression. Unpublished doctoral dissertation.. Southern Cross University, NSW Australia.

Wang, K., \& Hermann, C. (2006). Pilot study to test the effectiveness of healing touch on agitation in people with dementia. Geriatric Nursing, 27(1), 34-40.

Ward, R. P., Kugelmas, M., \& Libsch, K. D. (2004). Management of hepatitis C: Evaluating suitability for drug therapy. American Family Physician, 69(6), 14291436.

Wardell, D. W., \& Weymouth, K. F. (2004). Review of studies of healing touch. Journal of Nursing Scholarship, 36(2), 147-154. 
Wardell, D. (2005). Healing touch for chronic neuropathic pain,. International Journal of Human Caring, 9(2), 123-124.

Wheeler-Robins, J. L. (1999). Psychoneuroimmunology and healing touch in HIV disease. Unpublished doctoral dissertation. Virginia Commonwealth University, Virginia, Dissertation Abstracts International, 61, 01B.

White, C. P, Hirsch, G., Patel, S., Adams, F., \& Peltekian, K. M. (2007) Complementary and alternative therapy use by patients chronically infected with hepatitis $\mathrm{C}$ virus. Canadian Journal of Gastroenterology. 2I(9), 589-595.

Wilkinson, D. S., Knox, P. L., Chatman, J. E., Johnson, T. L., Barbour, N., Myles, Y., et al.. (2002). The clinical effectiveness of healing touch. Journal of Alternative and Complementary Medicine, 8(1), 33-47.

Winstead-Fry, P., \& Kijek, K. (1999). An integrative review and meta-analysis of therapeutic touch research Alternative Therapies. 5, 58-67.

Zic, I. (2005). Peginterferon alpha/ribavirin combination therapy for the treatment of hepatitis C infection. Gastroenterology Nursing, 28(4) 317-328.

Zichmond, S. L., Bryce, C. L.,Blasiole, J. A., Shinkunas, L., LaBrecque, D. R., \& Arnold, R. M. (2006). Majority of patients with hepatitis $\mathrm{C}$ express physical, mental and social difficulties with antiviral treatment. European Journal of Gastroenterology and Hepatology. 18(4) 381-388.

Zucker, D. M. (2001). An exercise intervention to prevent hepatitis related fatigue. Hepatology, 4(4). 
Appendix A

Demographic Data Form

Date:

Code:

1. Age in years

2. Gender

a) Male

b) Female

3. Education Level
a) Less than secondary
b) Secondary
c) Some post-secondary
d) University graduate

4. Race
a) African Canadian
b) Aboriginal
c) Asian/Pacific Islander
d) Caucasian
e) Hispanic
f) Multiple Race/Other

5. Marital Status
a) Single/never married
b) Married/common-law
c) Divorced/separated
d) Widowed

6. Occupation when employed

7. Annual Household Income Ranges

a) Less than $\$ 30,000$

b) Between $\$ 30,000$ and $\$ 44,999$

c) Between $\$ 45,000$ and $\$ 59,000$

d) $\$ 60,000$ or more.

8. Year of Diagnosis of $\mathrm{HCV}+$

9. Genotype, if known

10. Month/Year started Interferon therapy

11. Other diagnoses

12. Current medications taken 


\section{Appendix B}

Healing Touch Magnetic Clearing Intervention

Preparation prior to the intervention takes five minutes. The practitioner greets the client using a written script that explains the session will last for 30 minutes and will involve the use of hand motions above the client to facilitate relaxation. The intervention is provided with the subject clothed. The practitioner instructs the client to remove his shoes and glasses (if desired) and lie on his back on the table. The practitioner offers to cover the client with a sheet and to place a bolster under his knees for comfort. The practitioner will inform the client he can keep his eyes open or closed and lie quietly during the session. The practitioner begins by standing quietly next to the client and consciously centering. Centering is being fully present in the moment, letting go of outside distractions and connecting to one's inner self. The practitioner then shifts their focus and attunes to the client. Attunement is consciously setting the intention to enter into an energetic relationship with the client. The practitioner does an energetic assessment by passing their hands from the client's head to feet above the body noticing any sensation they feel in their palms. Next the technique is provided without touching the client.

1. The practitioner begins by standing at the client's right side and placing both hands 12 inches above the top of the client's crown. All fingers are spread, relaxed and slightly curved with the thumbs close to each other.

2. Using a long continuous combing or raking motion the practitioner will maintain the hand position moving down the middle of the client's entire body finishing below the feet. 
3. The practitioner's hand movement from above the head to below the feet is one smooth, continuous, uninterrupted motion. Hands are held approximately six inches off the body.

4. The time from start to finish of each sweep down the body is 30 seconds.If the practitioner has a sense of energy build up in their hands they can shake them gently after completing the sweep at the client's feet.

5. After ten sweeps down the middle of the body the practitioner will move to the left and sweep the left side of the body from above the head to below the feet using the same hand position and at the rate of 30 seconds for ten more sweeps.

6. The practitioner then moves back to the client's right side and using the same hand movement provides ten more sweeps on the right side in the same manner.

7. Once the practitioner completes a total of 30 sweeps down the client's body the technique is completed.

8. The practitioner stands or sits in silence for five minutes while remaining focused on the client.

9. To complete the session the practitioner informs the client the session is finished. The practitioner slowly assists the client to a sitting position on the table allowing his feet to dangle over the side for a few minutes and instructing him to stretch his fingers and toes.

10. The practitioner is responsible to make sure the client is mentally alert before discharging them from the session. 


\section{Appendix C}

\section{Mock Touch Intervention}

The volunteer greets the subject using a written script that explains the entire session will last for 30 minutes and will involve using hand motions beside the client. The intervention is provided with the subject clothed. The volunteer instructs the client to remove his shoes and glasses (if desired) and lie on his back on the table. The volunteer offers to cover the client with a sheet and to place a bolster under his knees for comfort. The volunteer will inform the client he can keep his eyes open or closed and should just lie quietly during the session. The volunteer then stands in silence next to the client with the intention of not connecting with the client and starts silently counting backwards from 100 by serial 7 to occupy their mind. During this time the volunteer will gently shift their weight from one foot to the other. The intervention will be provided without touch.

1. The volunteer begins by standing at the client's right side and placing both hands with the palms facing floor at the level of the subject's neck. All fingers are spread, relaxed and straight with the thumbs close to each other.

2. Using an up and down wavy motion the volunteer will maintain the hand position moving down the length of the body to the knees.

3. The volunteer's hand movement from neck to the knees is at least 12 inches from the body.

4. The time for each brush down the body is 30 seconds from start to finish. The volunteer continues to engage their mind by counting backward with serial sevens and intentionally not connecting with the client. 
5. After fifteen repeats beside the right of the body the volunteer will move to the left and brush beside the left side of the body from neck to below the knees using the same hand position and at the same rate of 30 seconds for fifteen more times.

6. Once the volunteer completes a total of 30 hand movements in the air next to client's body the technique is completed.

7. The volunteer stands or sits in silence for five minutes while remaining focused on counting backward by serial sevens.

8. To complete the session the volunteer will tell the client the session is complete. The practitioner slowly assists the volunteer to a sitting position on the table allowing his feet to dangle over the side for a few minutes and instructs him to stretch his fingers and toes.

9. The volunteer is responsible to make sure the client is mentally alert before releasing the client from the session. 


\section{Appendix D}

\section{Schwartz Cancer Fatigue Scale -6}

The words and phrases below describe different feelings people associate with fatigue. Please read each item and circle the number that indicates how much fatigue has made you feel in the past 2 to 3 days.

$$
\begin{aligned}
& 1=\underline{\text { not at all }} \\
& 2=\underline{\text { a little }} \\
& 3=\underline{\text { moderately }} \\
& 4=\text { quite a bit } \\
& 5=\underline{\text { extremely }}
\end{aligned}
$$

\begin{tabular}{|c|c|c|c|}
\hline Tired ......................... & 1 & 2 & \\
\hline Difficulty thinking ......... & 1 & 2 & \\
\hline Overcome................... & 1 & 2 & \\
\hline Listless ...................... & 1 & 2 & \\
\hline Worn out .................... & 1 & 2 & \\
\hline Helpless. & 1 & 2 & \\
\hline
\end{tabular}

C(C1997 A. L. Schwartz. Permission is granted for use with appropriate acknowledgment. 
Appendix E

Subject's Co- morbidities, Hemoglobin Level and Current Meds

\begin{tabular}{|c|c|c|c|}
\hline Subject & Co-morbidities & Hemoglobin & Current Meds \\
\hline Subject 1 & $\begin{array}{l}\text { Previous MI, } \\
\text { Hypertension }\end{array}$ & 15.6 & Folic Acid \\
\hline Subject 2 & $\begin{array}{l}\text { Diabetes Mellitus } \\
\text { Hemochromotosis } \\
\text { Hypertension }\end{array}$ & 12.7 & $\begin{array}{l}\text { Hyzaar } \\
\text { Nexium } \\
\text { Tylenol } \\
\text { Folic Acid }\end{array}$ \\
\hline Subject 3 & Arthritis & 12.6 & Ativan \\
\hline Subject 4 & Spinal Stenosis & 10.4 & $\begin{array}{l}\text { Folic Acid } \\
\text { Tyenol }\end{array}$ \\
\hline Subject 5 & Alopecia & 18.0 & None \\
\hline Subject 6 & $\begin{array}{l}\text { Insomnia } \\
\text { Arthritis } \\
\text { Depression }\end{array}$ & 15.3 & $\begin{array}{l}\text { Celexa } \\
\text { Oxcyepan } \\
\text { URSO }\end{array}$ \\
\hline Subject 7 & Depression & 12.0 & $\begin{array}{l}\text { Synthroid } \\
\text { Folic Acid } \\
\text { Iron supplement }\end{array}$ \\
\hline Subject 8 & $\begin{array}{l}\text { High Iron } \\
\text { High Blood Pressure } \\
\text { Obesity } \\
\text { Patchy Liver Steatosis } \\
\text { Porphyria cutanea tarda }\end{array}$ & Not available & $\begin{array}{l}\text { Loversyl } \\
\text { Tylenol }\end{array}$ \\
\hline Subject 9 & $\begin{array}{l}\text { Hypothyroidism } \\
\text { L ventricular hypertrophy } \\
\text { Urinary Tract Infection }\end{array}$ & 12.6 & $\begin{array}{l}\text { Synthroid } \\
\text { Tylenol }\end{array}$ \\
\hline Subject 10 & Prostatitis & Not available & $\begin{array}{l}\text { Losec } \\
\text { Zopiclone } \\
\text { Tylenol } \\
\text { Ativan }\end{array}$ \\
\hline
\end{tabular}


Subject Co-morbidities Hemoglobin Current Meds

Subject 11 Anxiety

Depression

Hypothyroid

Subject 12 Asthma

Depression

Psoriasis

Subject 13 Migraines

Subject 14

Anxiety Disorder

Arthritis

Asthma

Depression

Hypertension

Subject 15 None

Subject 16 None

Subject 17 Rheumatoid Arthritis
16.2

Mirtazapine

Atrovent

Flovent

Rantidine

Synthroid

Trazadene

11.6

Ativan

Effexor XR

Trazadone

Milk Thistle

Grapefruit extract

Vitamin B complex

Vitamin $\mathrm{C}$ and $\mathrm{E}$

12.0

Folic Acid

Calcium

Tylenol

Vitamin A, C, D, E

Ground Flax Seed

13.8 Effexor

Amitryptalin

Bricanyl

Elavil

$14.8 \quad$ None

14.8 Ester C

Nutra Sea PA

Magneain

Mellaril

Not available Folic Acid

Milk Thistle 


\section{Capital Health Appendix G}

Research Ethics Board

5790 University Avenue

Room 118, Centre for Clinical Research

Halifax, NS B3H 1 V7

Phone: 473-5726

Fax: 473-5620

June 292006

Ms. Ann Taylor

1346 Dresden Row

Halifax, NS B3J $2 \mathrm{J8}$

Dear Ms. Taylor

Full Approval

(June 19, 2006 - June 19, 2007)

\section{RE: The Use of Healing Touch in Patients with Hepatitus C Virus Receiving Interferon Therapy}

REB File Number: CDHA-RS/2006-176

Thank you for responding to the concerns of the Research Ethics Board and for forwarding a copy of the clarifications requested regarding this protocol.

Documents resubmitted for review included:

- Response Letter (dated June 27, 2006)

- Consent to Take Part in a Clinical Trial - Patient Information (Version 1.1; dated June 27, 2006)

- Ethics Approval Submission Form

- Appendix A - Demographic Data Form (Version 1.0;, dated May 15, 2006)

- The Medical Algorithms Project - Fatigue Impact Scale and CES-D Self-Report Depression Scale

I have reviewed the above noted documents and note that all requested protocol clarifications and consent form modifications have been completed. I am now pleased to confirm the Board's full approval for this research submission at Capital Health.

This includes approval for the following documents:

- Consent to Take Part in a Clinical Trial - Patient Information (Version 1.1; dated June 27, 2006)

- Research Protocol HCV and Fatigue (Version 1; dated May 15, 2006)

- Healing Touch International, Inc. Spreading Healing Light Worldwide - International Code of Ethics for Healing Touch Practitioners (11/25/02)

$$
\text { MA }
$$


Approval by the Research Ethics Board is for scientific validity and ethical acceptability; it does not include any administrative considerations for the use of hospital resources. A copy of your submission has been forwarded to the Centre for Clinical Research; they will discuss any resource requirements with you.

The Research Ethics Board for the Capital District Health Authority complies with the Tri-Council Policy Statement, the ICH Harmonized Tripartite Guidelines: Good Clinical Practice, and Division 5 of the Food and Drug Regulations.

The Board would remind you that, in accordance with ethical guidelines, once a study has been approved, the investigator assumes responsibility to submit an annual progress report on the anniversary date (June 19).

If you do not have your Annual Approval approved prior to the Anniversary Date you are working outside the approval of the Capital Health Research Ethics Board and the study is subject to suspension.

The Board should also be made aware of any:

- Serious adverse events.

- Changes to the initial submission or closure of the study within 90 days of the event.

- Should any material be designed for advertisement or publication with a view to attracting patients, the Research Ethics Board should review it first.

- Approval studies may be audited, should your research be selected for audit, the Board will advise you and indicate any other requests at that time.

This letter is in lieu of the Health Canada Research Ethics Board Attestation Form.

For future correspondence concerning this project, you must refer to the Research Ethics Board assigned file number (CDHA-RS/2006-176).
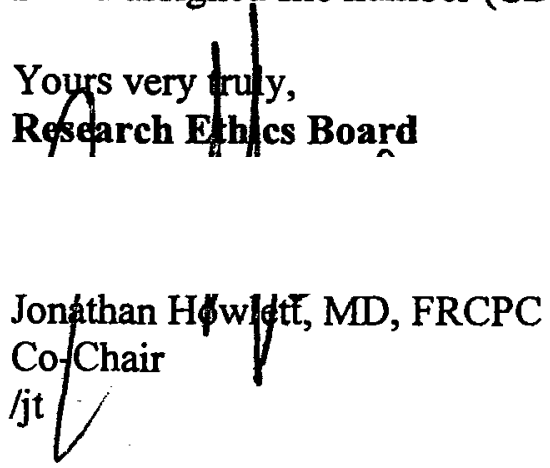

CC: Dr. Kevork Peltekian 